ASI-TPA/14/95 SPhT 95/118 hep-th/9510175

\title{
From CFT to Graphs
}

\author{
V.B. Petkova \\ Arnold Sommerfeld Institute for Mathematical Physics, \\ TU Clausthal, Leibnizstr. 10, D-38678 Clausthal-Zellerfeld, Germany, \\ Institute for Nuclear Research and Nuclear Energy ${ }^{\dagger}$, \\ Tzarigradsko Chaussee 72, 1784 Sofia, Bulgaria \\ and \\ J.-B. Zuber \\ CEA, Service de Physique Théorique de Saclay *, \\ F-91191 Gif sur Yvette Cedex, France
}

In this paper, we pursue the discussion of the connections between rational conformal field theories (CFT) and graphs. We generalize our recent work on the relations of operator product algebra (OPA) structure constants of $s l(2)$ theories with the Pasquier algebra attached to the graph. We show that in a variety of CFT built on $\operatorname{sl}(n)$ - typically conformal embeddings and orbifolds, similar considerations enable one to write a linear system satisfied by the matrix elements of the Pasquier algebra in terms of conformal data - quantum dimensions and fusion coefficients. In some cases, this provides a sufficient information for the determination of all the eigenvectors of an adjacency matrix, and hence of a graph.

10/95 submitted to Nuclear Physics B

$\dagger$ Permanent address.

* Laboratoire de la Direction des Sciences de la Matière du Commissariat à l'Energie Atomique. 


\section{Introduction}

In this paper we explore further the connections between rational conformal field theories and graphs. The idea inherited from the earlier work of Pasquier [1] is that there are tight connections between some features of rational conformal field theories based on $s l(n)$, typically WZW models and the corresponding minimal cosets, on the one hand, and generalized lattice "height" models based on graphs, on the other. The most conspicuous of these connections lies in the spectral properties of the two kinds of theories: the same $s l(n)$ weights that label the spin zero fields of the CFT also label the eigenvalues of the adjacency matrices of the graphs [2].

This connection has been strengthened by the recent observation [3] that it extends to the level of operator algebras: in $s l(2)$ theories, the ratios of structure constants of the OPA of spin zero fields pertaining to a pair of CFT's of identical level are given by the structure constants $M_{\lambda \mu}{ }^{\nu}$ of an algebra intrinsically attached to the graph. This algebra, that we call the Pasquier algebra, has structure constants given by a Verlinde-like formula, in which the components of the eigenvectors $\psi$ of the adjacency matrix of the graph replace the modular $S$ matrix: see below for a more precise expression.

These considerations assumed the knowledge of the graph. In [2], graphs relevant for the case of $s l(3)$ were found by empiric ways, essentially by trials and errors. The purpose of this paper is to show that, at least in a restricted class of CFT with an extended chiral symmetry algebra, the previous connection between the structure constants and the Pasquier algebra may be inverted. Data of the CFT enable one to determine the structure constants $M$ of the Pasquier algebra, the diagonalization of which yields the eigenvectors $\psi$ and thus the adjacency matrix and the graph.

This paper is organized as follows: after introducing in sect. 2 some notations and some basic properties of the graphs, we shall study in sect. 3 the operator algebra of conformal theories associated with conformal embeddings and propose an equation that connects conformal and graphical data. This will be probed in sect. 4 on various examples. Section 5 uses the theory of " $C$-algebras" to obtain more explicit relations on eigenvectors of the adjacency matrices. Two appendices present one further example and a discussion of the $\operatorname{sl}(3)$ block-diagonal $D$ - series.

\section{Notations}

This section is devoted to a summary of notations and concepts introduced in our former work. 


\subsection{On CFT's}

Working with the $\operatorname{sl}(n)$ algebra and its affine extension $\widehat{s l}(n)_{k}$, at a certain integer level $k$, we first introduce some Lie algebraic objects. Let $\hat{\Lambda}_{1}, \cdots, \hat{\Lambda}_{n-1}$ be the fundamental weights of $\operatorname{sl}(n)$. (The hat is intended to distinguish them from the later notation $\Lambda$ for a pair of weights.) Let $\rho=\hat{\Lambda}_{1}+\cdots+\hat{\Lambda}_{n-1}$ be the sum of these fundamental weights. Since in a WZW theory, the weight $\rho$ labels the identity field, we shall also occasionally denote it by $\mathbf{1}$. The set of integrable weights (shifted by $\rho$ ) of the affine algebra $\widehat{s l}(n)_{k}$ is

$$
\mathcal{P}_{++}^{(k+n)}=\left\{\lambda=\lambda_{1} \hat{\Lambda}_{1}+\cdots+\lambda_{n-1} \hat{\Lambda}_{n-1} \mid \lambda_{i} \in \mathbb{Z}, \quad \lambda_{i} \geq 1, \quad \sum_{i} \lambda_{i} \leq k+n-1\right\} .
$$

We shall encounter the $\mathbb{Z}_{n}$ group of automorphisms of this set of weights, generated by

$$
\sigma: \lambda=\left(\lambda_{1}, \lambda_{2}, \cdots, \lambda_{n-1}\right) \mapsto \sigma(\lambda)=\left(n+k-\lambda_{1}-\cdots-\lambda_{n-1}, \lambda_{1}, \cdots, \lambda_{n-2}\right) .
$$

We also make use of the $n$ (linearly dependent) vectors $e_{i}$

$$
e_{1}=\hat{\Lambda}_{1}, \quad e_{i}=\hat{\Lambda}_{i}-\hat{\Lambda}_{i-1}, \quad i=2, \cdots, n-1, \quad e_{n}=-\hat{\Lambda}_{n-1}
$$

(the weights of the fundamental representation of highest weight $\hat{\Lambda}_{1}$ ) and of the symmetric bilinear form on weight space

$$
\left(e_{i}, e_{j}\right)=\delta_{i j}-\frac{1}{n} .
$$

We shall be mainly considering the WZW model with a current algebra $\widehat{s l}(n)_{k}$, or the simplest coset CFT's

$$
\frac{\widehat{s l}(n)_{k-1} \times \widehat{s l}(n)_{1}}{\widehat{s l}(n)_{k}}
$$

also called the "minimal $W_{n}$ models". In the WZW theories, primary fields are labelled by a pair of integrable weights at level $k$, and this pair is denoted by a capital $\Lambda$

$$
\Lambda=(\lambda, \bar{\lambda}), \quad \lambda, \bar{\lambda} \in \mathcal{P}_{++}^{(k+n)} .
$$

In the coset theories, we rather need two such pairs at level $k$ and $k-1$ 且

$$
\left((\lambda, \bar{\lambda}),\left(\lambda^{\prime}, \bar{\lambda}^{\prime}\right)\right), \quad \lambda, \bar{\lambda} \in \mathcal{P}_{++}^{(k+n)}, \quad \lambda^{\prime}, \bar{\lambda}^{\prime} \in \mathcal{P}_{++}^{(k+n-1)}
$$

1 In principle we should use three pairs of weights

$$
\left((\lambda, \bar{\lambda}),\left(\lambda^{\prime}, \bar{\lambda}^{\prime}\right),\left(\lambda^{\prime \prime}, \bar{\lambda}^{\prime \prime}\right)\right), \quad \lambda, \bar{\lambda} \in \mathcal{P}_{++}^{(n+k)}, \quad \lambda^{\prime}, \bar{\lambda}^{\prime} \in \mathcal{P}_{++}^{(n+k-1)}, \quad \lambda^{\prime \prime}, \bar{\lambda}^{\prime \prime} \in \mathcal{P}_{++}^{(n+1)},
$$

However we can make use of the $\mathbb{Z}_{n}$ automorphism $\sigma$ of (2.2), and of the fact that in the coset theory the pairs $\left(\lambda, \lambda^{\prime}\right)$ and $\left(\bar{\lambda}, \bar{\lambda}^{\prime}\right)$ are determined modulo a diagonal action of $\sigma$, to rotate $\lambda^{\prime \prime}, \bar{\lambda}^{\prime \prime}$ to the standard value $\rho$. 
In fact, as in [3], we shall concentrate on the "thermal" fields, for which $\lambda^{\prime}=\bar{\lambda}^{\prime}=\rho$, and thus label simply these fields by the same notation $\Lambda$ as in (2.5).

As in any CFT, the way the left and right representations of the chiral algebra (affine or Virasoro or more generally $W_{n}$ ) are coupled to produce the primary fields of the theory is encoded in the genus one partition function

$$
Z=\sum_{\lambda, \bar{\lambda}} \mathcal{N}_{\lambda \bar{\lambda}} \chi_{\lambda}(q) \chi_{\bar{\lambda}}(\bar{q})
$$

Classification of such modular invariant partition functions has been accomplished only in a few cases, [4 6]. In eq. (2.6), we have made use of notations relevant for affine algebras. For coset theories, it is clear that a large class of solutions is obtained through the factorization of the matrix $\mathcal{N}$ into those of the factors of the coset

$$
\mathcal{N}_{\left(\lambda, \lambda^{\prime}\right),\left(\bar{\lambda}, \bar{\lambda}^{\prime}\right)}^{\text {coset }}=\mathcal{N}_{\lambda \bar{\lambda}}^{(k)} \mathcal{N}_{\lambda^{\prime} \bar{\lambda}^{\prime}}^{(k-1)}
$$

(see [7] for a thorough discussion of all the possible solutions) but we shall consider solely the cases in which the level $(k-1)$ invariant is "trivial"

$$
\mathcal{N}_{\left(\lambda, \lambda^{\prime}\right),\left(\bar{\lambda}, \bar{\lambda}^{\prime}\right)}^{\text {coset }}=\mathcal{N}_{\lambda \bar{\lambda}}^{(k)} \delta_{\lambda^{\prime} \bar{\lambda}^{\prime}}
$$

In general, we find it appropriate to distinguish between the two cases of:

* Type I theories for which the partition function (2.6) may be recast as a sum of squares of sums of characters

$$
Z=\sum_{i}\left|\sum_{\lambda \in \mathcal{B}_{i}} \chi_{\lambda}\right|^{2} .
$$

* Type II theories for which this rewriting is impossible without the introduction of signs

$$
Z=\sum_{i} \pm\left|\sum_{\lambda \in \mathcal{B}_{i}} \chi_{\lambda}\right|^{2} .
$$

The former case signals the existence of an extended chiral algebra, generated by holomorphic fields that appear in the block of the identity, and whose irreducible representations labelled $\mathcal{B}_{i}$ decompose into irreducible representations of the current algebra as shown in the expression of $Z$. $⿴$ The latter cases, (that concern us less in the present paper, are

2 Some representations $\lambda$ may appear in $\mathcal{B}_{i}$ with a nontrivial multiplicity $\operatorname{mult}_{\mathcal{B}_{i}}(\lambda) \in \mathbb{N}$, i.e., in (2.9) $\chi_{\mathcal{B}_{i}}=\sum_{\lambda \in \mathcal{P}_{++}} \operatorname{mult}_{\mathcal{B}_{i}}(\lambda) \chi_{\lambda}$. 
known to be related to some of the former by a twist of the right sector with respect to the left one according to an automorphism of the fusion rules [8 10].

In many of the subsequent considerations, we shall be discussing spin zero fields, for which the left and right components are identical $\lambda=\bar{\lambda}$. For those fields, we shall adopt a shorthand notation, using $\lambda$ instead of $\Lambda=(\lambda, \lambda)$. We do of course the same for chiral quantities, i.e. quantities intrinsically attached to a single component. This is in particular the case of the fusion algebra whose coefficients are given by the celebrated Verlinde formula [11]

$$
N_{\lambda \mu}{ }^{\nu}=\sum_{\sigma} \frac{S_{\lambda \sigma} S_{\mu \sigma} S_{\nu \sigma}^{*}}{S_{1 \sigma}} .
$$

In contrast, coefficients, or structure constants, of the operator product algebra (OPA) are not chiral quantities and depend in an essential way on the coupling between the left and right sectors.

\subsection{On Graphs}

The second category of objects that we shall be handling are graphs. When dealing with $\operatorname{sl}(n)$ theories, we postulate that each of these graphs $\mathcal{G}$ satisfies the following requirements:

i) it is connected;

ii) it is symmetric, i.e. unoriented;

iii) let $\mathcal{V}$ be the set of vertices; to each $a \in \mathcal{V}$ may be attached a $\mathbb{Z} / n \mathbb{Z}$ grading $\tau(a)$, the " $n$-ality", and the only nonvanishing entries of the adjacency matrix $G$ (i.e. the only edges) are between vertices of different $\tau$. This enables one to split this adjacency matrix into a sum of $n-1$ matrices

$$
G=G_{1}+G_{2}+\cdots+G_{n-1}
$$

where $G_{p}$ is the adjacency matrix describing the edges that connect vertices of $n$-ality differing by $p$

$$
\left(G_{p}\right)_{a b} \neq 0 \quad \text { only if } \quad \tau(b)=\tau(a)+p \bmod n
$$

Accordingly, the graph may be regarded as the superposition on the same set of vertices of $n-1$ oriented (except for $p=n / 2$ ), not all necessarily connected, graphs $\mathcal{G}_{p}$ of adjacency matrices $G_{p}, p=1, \cdots, n-1 ;$

iv) there exists an involution $a \mapsto a^{\vee}$ on $\mathcal{V}$ such that $\tau\left(a^{\vee}\right)=-\tau(a)$ and

$$
\left(G_{p}\right)_{a b}=\left(G_{p}\right)_{b^{\vee} a^{\vee}}
$$


v) the matrices $G_{p}$ are pairwise transposed of one another:

$$
{ }^{t} G_{p}=G_{n-p}
$$

vi) the matrices $G_{p}$ commute among themselves, in particular each $G_{p}$ commutes with its transpose $G_{n-p}$, hence is "normal", i.e. diagonalizable in an orthonormal basis common to all of them;

vii) these common eigenvectors are labelled by integrable weights $\lambda \in \mathcal{P}_{++}^{(k+n)}$ for some level $k$, we denote them $\psi_{a}^{(\lambda)},(a \in \mathcal{V})$, and the corresponding eigenvalues of $G_{1}, G_{2}$, $\ldots, G_{n-1}$ are given by the following formulae

$$
\begin{aligned}
& \gamma_{1}^{(\lambda)}=\sum_{i=1}^{n} \exp -\frac{2 i \pi}{h}\left(e_{i}, \lambda\right) \\
& \gamma_{2}^{(\lambda)}=\sum_{1 \leq i<j \leq n} \exp -\frac{2 i \pi}{h}\left(\left(e_{i}+e_{j}\right), \lambda\right) \\
& \vdots \quad \vdots \quad \vdots \\
& \gamma_{n-1}^{(\lambda)}=\sum_{1 \leq i_{1}<\cdots i_{n-1} \leq n} \exp -\frac{2 i \pi}{h}\left(\left(e_{i_{1}}+\cdots+e_{i_{n-1}}\right), \lambda\right), \\
& \gamma_{n-p}^{(\lambda)}=\left(\gamma_{p}^{(\lambda)}\right)^{*}=\gamma_{p}^{\left(\lambda^{*}\right)},
\end{aligned}
$$

where $h=k+n$ and $\lambda^{*}$ is the conjugate weight $\left(\lambda_{n-1}, \ldots, \lambda_{1}\right)$; some of these $\lambda$ may occur with multiplicities larger than one;

viii) $\rho=(1,1, \cdots, 1)$ is among these $\lambda$, with multiplicity 1 : it corresponds to the eigenvector of largest eigenvalue, $\gamma_{p}^{(\rho)} \geq\left|\gamma_{p}^{(\lambda)}\right|$, the so-called Perron-Frobenius eigenvector; its components $\psi_{a}^{(\rho)}, a \in \mathcal{V}$, are nonvanishing and positive;

ix) the graph $\mathcal{G}_{1}$ admits at least one extremal vertex, i.e. a vertex on which only one edge is ending and from which only one edge is starting. This vertex is denoted $\mathbf{1}$ and we put two additional requirements on it

1) $\mathbf{1}^{\vee}=\mathbf{1}$, and thus $\tau(\mathbf{1})=0,3$

2) all the components of the $\psi$ relative to this vertex are nonvanishing and positive: $\forall \lambda \quad \psi_{1}^{(\lambda)}>0$.

3 Although assumption 1) is not satisfied by all graphs that we know, it seems to be met by those that are relevant in the context of the present paper, namely those of type I. 
As a consequence of $\mathrm{ix}-1$ ), the two points connected to 1 are conjugate to one another under the involution and will be denoted respectively $a_{f}$ and $a_{f}^{\vee}$.

For the benefit of the reader who is not convinced by the naturalness of these requirements, we shall point out that the graphs $\mathcal{G}_{p}$, resp. their adjacency matrices $\left(G_{p}\right)_{a b}$, are natural extensions of the fusion graphs, resp. fusion coefficients of the $n-1$ fundamental representations of $\widehat{s l}(n)_{k}$; in that particular case, $\mathcal{V}=\mathcal{P}_{++}^{(k+n)}, \tau$ is the natural $\mathbb{Z} / n \mathbb{Z}$ grading of representations of $\operatorname{sl}(n)$ (number of boxes of the Young tableau modulo $n$ ), while the involution is the conjugation of representations; the commutation and spectral properties of the $G_{p}$ are also natural in that case, with the expressions (2.16) following from the Verlinde formula, together with the explicit form of the modular matrix $S$; in that particular case, $\psi \equiv S$.

In the case $n=2$, the only graphs satisfying these conditions are the $A D E$ Dynkin diagrams [12] and the weights $\lambda$ labelling the eigenvalues are the Coxeter exponents. For $n=3$, some graphs have been found satisfying these conditions, see [2]. By extension of the $\operatorname{sl}(2)$ situation, we call again "exponents" the corresponding $\lambda$ and denote their set by Exp. It is a simple consequence of eq. (2.13) that the set Exp is invariant under the automorphism (2.2) and that the eigenvectors may be chosen so as to satisfy

$$
\psi_{a}^{(\sigma(\lambda))}=e^{2 i \pi \frac{\tau(a)}{n}} \psi_{a}^{(\lambda)}
$$

These graphs are not, however, in general, fusion graphs. This is evidenced if out of the set of eigenvectors $\psi_{a}^{(\lambda)}$ and their complex conjugates $\psi_{a}^{(\lambda) *}$, satisfying

$$
\begin{gathered}
\sum_{a \in \mathcal{V}} \psi_{a}^{(\lambda)} \psi_{a}^{(\nu) *}=\delta_{\lambda \nu}, \quad \sum_{\lambda \in \operatorname{Exp}} \psi_{a}^{(\lambda)} \psi_{c}^{(\lambda) *}=\delta_{a c} \\
\psi_{a^{\vee}}^{(\lambda)}=\psi_{a}^{(\lambda) *}=\psi_{a}^{\left(\lambda^{*}\right)}
\end{gathered}
$$

one forms the following two sets of real numbers

$$
\begin{aligned}
M_{\lambda \mu}{ }^{\nu} & =\sum_{a \in \mathcal{V}} \frac{\psi_{a}^{(\lambda)} \psi_{a}^{(\mu)} \psi_{a}^{(\nu) *}}{\psi_{a}^{(\rho)}} \\
N_{a b}{ }^{c} & =\sum_{\lambda \in \operatorname{Exp}} \frac{\psi_{a}^{(\lambda)} \psi_{b}^{(\lambda)} \psi_{c}^{(\lambda) *}}{\psi_{1}^{(\lambda)}} .
\end{aligned}
$$

These $M$ 's and $N$ 's may be regarded as the structure constants of two commutative and associative algebras, dual to one another. They are two generalizations of the Verlinde 
formula to which they both reduce in the self-dual case where $\psi=S$. Beside these selfdual cases, the $M$ 's are in general not integers but rather algebraic numbers, belonging to the number field generated by the $\psi$ 's. It is therefore surprising that in contrast, the $N$ 's are still found to be rational numbers, and in fact in most known examples they are integers. More precisely, there exists at least one choice of the vertex "1", and if some of the eigenvalues are degenerate, one choice of the basis $\psi$ such that the $N$ 's be rational integers. This is an empirical observation for which no general proof is known to us. Note that we can identify the graph (that is the set of vertices and the collection of arrows associated with any $G_{p}$ ) with the set of matrices $\left\{N_{b}\right\}$ and the action of any $G_{p}$ defined as the (left) matrix multiplication. Indeed we have

$$
G_{p} N_{a}=\sum_{b}\left(G_{p}\right)_{a b} N_{b}
$$

which is obtained inserting the r.h.s. of (2.19b) for $\left(N_{a}\right)_{b}^{c}=N_{a b}^{c}$, exploiting also the fact that $G_{p}$ is diagonalised by the same eigenvectors, i.e., $\left(G_{p}\right)_{b c}=\sum_{\lambda \in \operatorname{Exp}} \gamma_{p}^{(\lambda)} \psi_{b}^{(\lambda)} \psi_{c}^{(\lambda) *}$, and using the first orthogonality relation in (2.18a). Taking in the l.h.s of (2.20) the identity matrix $N_{\mathbf{1}}$ we see that the $G_{p}$ belong to the algebra of $N$ matrices and are in fact linear combinations of these $N$ matrices with non negative integral coefficients. Also it is easy to prove that the $N$ algebra is $\mathbb{Z}_{n}$ graded i.e. that $N_{a b}^{c} \neq 0$ only if $\tau(c)=\tau(a)+\tau(b) \bmod n$ as a consequence of the invariance of the set Exp under $\sigma$ and of the transformation (2.17) of the eigenvectors. In particular $G_{1}$ and $G_{n-1}$ coincide with the matrices $N_{a_{f}}$ and $N_{a_{f}^{\vee}}$ respectively because of ix) above. The fact that the adjacency matrices are found within the $N$ algebra will be used below.

These graphs are relevant for two related problems. First, they are believed to allow the construction of generalized height (or RSOS) integrable and critical lattice models. This is true for $n=2$ [1] and has been verified also in some cases of $n=3$ [2, 13], but it is likely that the previous conditions are not restrictive enough in general. On the other hand these lattice models are related to the previous topic of $s l(n)$ CFT's insofar as their continuous limit is described by the minimal coset above. Again, this is convincingly demonstrated only in the case of $s l(2)$, whereas the other cases rely on several empirical evidences.

i) The first of these evidences is that the same set of integrable weights of $\widehat{s l}(n)$ at level $k$ that label the diagonal terms of a modular invariant partition function (2.6) also describes the spectrum of eigenvalues of one of the graphs $\mathcal{G}$. 
In fact it appears that not all modular invariant partition functions of $s l(n)$ WZW theories may be matched with a graph. For example, no graph satisfying the conditions above can have a spectrum Exp matching the diagonal terms of the infinite series of modular invariants of $\widehat{s l}(3)$ for a level $k$ not a multiple of 3 , [14

$$
Z=\sum_{\lambda \in Q \cap \mathcal{P}_{++}^{(n+k)}}\left|\chi_{\lambda}\right|^{2}+\sum_{\lambda \in \mathcal{P}_{++}^{(n+k)} \backslash Q} \chi_{\lambda} \chi_{\sigma^{k \tau(\lambda)} \lambda}^{*}, \quad k \geq 4
$$

where $Q$ is the root lattice of $s l(n)$. Indeed the only diagonal terms in (2.21) come from the first sum, and the set $Q \cap \mathcal{P}_{++}^{(n+k)}$ is clearly not invariant under the action of $\sigma$, in contradiction with a property of the sets Exp, see above. Another counterexample is provided by the conformal embedding of $\widehat{A_{8}}$ into $\widehat{E_{8}}$, both at level 1 . There is only one representation of $\widehat{E_{8}}$ of level one, that decomposes into three representations of $\widehat{A_{8}}$, v.i.z. $\rho, \lambda_{3}=\rho+\hat{\Lambda}_{3}$ and $\lambda_{6}=\rho+\hat{\Lambda}_{6}$ [15]. The partition function reads

$$
Z=\left|\chi_{\rho}+\chi_{\lambda_{3}}+\chi_{\lambda_{6}}\right|^{2}
$$

As there are only three contributing representations, a possible graph satisfying the axioms of sect. 2.2 must have three vertices and cannot satisfy (2.13) for $p=1$.

As far as we can see, there are two possibilities at this stage. One may try to relax some of the conditions that we have put on the graphs. For example, demanding that (2.13) holds in a weaker form, with $\bmod n$ replaced in the r.h.s. by $\bmod m$, where $m$ is a divisor of $n$, would allow to accomodate the case (2.22) above, in which $G_{1}=G_{4}=$ $G_{7}, G_{2}=G_{5}=G_{8}, G_{3}=G_{6}=\mathbf{1}$, and $m=3$. Accordingly the set of exponents is invariant under the subgroup $\mathbb{Z}_{3}=\left\{1, \sigma^{3}, \sigma^{6}\right\}$ of $\mathbb{Z}_{9}$. We shall not pursue this line here. Alternatively, we may decide to concentrate on those modular invariants that are relevant for the coset theories, see (2.8). It is known [16] that the only branching functions (or characters) of cosets $G \times G / G$ that do not vanish are those for which, with our notations of sect. 2.1, $\lambda-\lambda^{\prime}-\lambda^{\prime \prime}+\rho$ belongs to the root lattice $Q$. For a modular invariant of the form (2.8), this implies that $\lambda-\bar{\lambda}$ has to belong to $Q$, which is not the case for the two counterexamples above. This is this attitude, which is consistent with the general belief that the graphs are deeply connected with the lattice models, hence with the coset theories, that was adopted in [17]. 
ii) The second evidence was pointed out recently in the case of $\operatorname{sl}(2)$ theories [3]. If one computes the ratios of structure constants of spin zero fields in two theories of identical level, say for the $D$ or $E$ type of theory over the $A$ theory of same level, one finds that

$$
M_{\lambda \mu \nu}=\frac{D_{(\lambda \lambda)(\mu \mu)(\nu \nu)}^{(D) \text { or }(E)}}{D_{(\lambda \lambda)(\mu \mu)(\nu \nu)}^{(A)}}
$$

(The reader is referred to [3] for a precise definition of the constants $D$ ).

iii) The last evidence was presented in [17]. There it was shown that for a given graph and the corresponding modular invariant identified through the observation i) above, there is an empirical coincidence between the non-negativity of the $M_{\lambda \mu}{ }^{\nu}$ and $N_{a b}^{c}$ coefficients and the property of the corresponding modular invariant partition function to belong to type I defined in (2.9). Then it was shown that for those graphs that have the structure constants $N$ and $M$ non negative, there exists an algebraic way to determine the partition function of the corresponding CFT. This was based on the theory of " $C$-algebras" that will be reviewed and used in sect. 5 of the present paper.

In the present paper we want to extend the discussion of the second point to $\operatorname{sl}(n)$ theories, $n>2$ and show its relations with the last point.

\section{Duality equations and constraints on the $M$ algebra.}

Let us consider one of the euclidean 4-point functions

$$
\left\langle\Phi_{\Lambda_{1}^{*}}\left(x_{1}\right) \Phi_{\Lambda_{2}^{*}}\left(x_{2}\right) \Phi_{\Lambda_{1}}\left(x_{3}\right) \Phi_{\Lambda_{2}}\left(x_{4}\right)\right\rangle
$$

in the minimal $W_{n}$ theories [18]. Here as explained above $\Lambda_{i}=\left(\lambda_{i}, \bar{\lambda}_{i}\right)$, with $\lambda_{i}, \bar{\lambda}_{i} \in$ $\mathcal{P}_{++}^{(k+n)}$, label fields of the subalgebra of "thermal" fields. The label should furthermore include some index to distinguish fields with the same pair of $\operatorname{sl}(n)$ weights, but for the time being we shall exclude the cases with such degeneracies. Denote by $\triangle_{\lambda}=\triangle_{\lambda^{*}}, \triangle_{\bar{\lambda}}$ the conformal weights of the field $\Phi_{\Lambda}$ and hence by $s(\Lambda):=\triangle_{\lambda}-\triangle_{\bar{\lambda}} \in \mathbb{Z}$ its spin. The physical fields decompose into chiral vertex operators (see, e.g.[9])

$$
\Phi_{\Lambda_{1}}(z, \bar{z})\left|\Lambda_{2}\right\rangle=\sum_{\substack{\Lambda_{5} \\
t, \bar{t}}} d_{\Lambda_{1} \Lambda_{2}}^{\Lambda_{5}(t, \bar{t})}\left(\begin{array}{c}
\lambda_{5} \\
\lambda_{1} \lambda_{2}
\end{array}\right)_{t}(z) \otimes\left(\begin{array}{c}
\bar{\lambda}_{5} \\
\bar{\lambda}_{1} \bar{\lambda}_{2}
\end{array}\right)_{\bar{t}}(\bar{z})\left|\Lambda_{2}\right\rangle
$$

with structure constants $d_{\Lambda_{1} \Lambda_{2}}^{\Lambda_{5} ; A}(A=(t, \bar{t})), d_{\Lambda \mathbf{1}}^{\Lambda}=1$. The index $t=1,2, \ldots, N_{\lambda_{1} \lambda_{2}}^{\lambda_{5}}$, labels a basis in the space $V_{\lambda_{1} \lambda_{2}}^{\lambda_{5}}$ of chiral vertex operators. To simplify the notation this index 
is omitted whenever it takes only one value, e.g., $N_{\lambda \lambda^{*}}^{\mathbf{1}}=N_{\lambda \mathbf{1}}^{\lambda}=1 ; N_{\lambda_{1} \lambda_{2}}^{\lambda_{3}}=\operatorname{dim} V_{\lambda_{1} \lambda_{2}}^{\lambda_{5}}$ is the Verlinde multiplicity (2.11),

$$
N_{\lambda_{1} \lambda_{2}}^{\lambda_{3}}=N_{\lambda_{2} \lambda_{1}}^{\lambda_{3}}=N_{\lambda_{1}^{*} \lambda_{2}^{*}}^{\lambda^{*}}=N_{\lambda_{1} \lambda_{3}^{*}}^{\lambda_{2}^{*}}
$$

The requirement of locality of the physical correlation functions leads, taking into account the braiding properties of the chiral vertex operators, to a set of equations for the structure constants $d_{\Lambda_{1} \Lambda_{2}}^{\Lambda_{5}}$. In particular exchanging the two middle fields and selecting the contribution of the identity $\lambda_{6}=\bar{\lambda}_{6}=\mathbf{1}=\rho$ in the intermediate channel we have

$$
\sum_{\substack{\Lambda_{5} \\ A, B}}(-1)^{s\left(\Lambda_{1}\right)+s\left(\Lambda_{5}\right)} d_{\Lambda_{2}^{*} \Lambda_{5}}^{\Lambda_{1} ; B} d_{\Lambda_{1} \Lambda_{2}}^{\Lambda_{5} ; A} F_{\lambda_{5},(r, t) ; \mathbf{1}}^{\lambda_{1}, \lambda_{2}} \bar{F}_{\bar{\lambda}_{5},(\bar{r}, \bar{t}) ; \mathbf{1}}^{\bar{\lambda}_{1}, \bar{\lambda}_{2}}=(-1)^{s\left(\Lambda_{2}\right)} d_{\Lambda_{1} \mathbf{1}}^{\Lambda_{1}} d_{\Lambda_{2}^{*} \Lambda_{2}}^{1}
$$

In (3.3) $F(\bar{F})$ denote particular elements of the left (right) fusion matrix $F_{\lambda_{5},(r, t) ; \lambda_{6},(s, u)}^{\lambda_{1}, \lambda_{2}}$, namely those for $\lambda_{6}=\mathbf{1}$. Upon suitable normalisation, such that the diagonal structure constants $\left(\Lambda_{i}=\left(\lambda_{i}, \lambda_{i}\right)\right)$ are given by $d_{\Lambda_{1} \Lambda_{2}}^{\Lambda_{5} ;(t, \bar{t})}=\delta_{t, \bar{t}}$, we can choose, whenever $N_{\lambda_{1} \lambda_{2}}^{\lambda_{5}} \neq 0$,

$$
F_{\lambda_{5},(r, t) ; \mathbf{1}}^{\lambda_{1}, \lambda_{2}}=\delta_{\sigma_{123}(r), t^{*}} F_{\lambda_{1} \lambda_{2}}^{\lambda_{5}}:=\delta_{\sigma_{123}(r), t^{*}} \sqrt{\frac{D_{\lambda_{5}}}{D_{\lambda_{1}} D_{\lambda_{2}}}}
$$

Here $D_{\lambda}$ is the quantum dimension, defined by a ratio of $\widehat{s l}(n)_{k}$ modular matrix elements, $D_{\lambda}=S_{\lambda \mathbf{1}} / S_{\mathbf{1 1}}$. In (3.4) $\sigma_{123}(r)$ is the image of the basis element in $V_{\lambda_{1} \lambda_{2}}^{\lambda_{5}}$ labelled by $r$ under the mapping $\sigma_{123} \equiv \sigma_{13} \sigma_{12}: V_{\mu \gamma}^{\lambda} \rightarrow \sigma_{13} V_{\lambda^{*} \gamma}^{\mu^{*}}=V_{\lambda^{*} \mu}^{\gamma *}$ and $t^{*}$ labels an element in $V_{\lambda_{1}^{*} \lambda_{2}^{*}}^{\lambda_{*}^{*}}$ with complex conjugated matrix elements (see [9]). From (3.4) and the fact that the quantum dimensions form a representation of the fusion algebra, it follows that

$$
\sum_{\lambda_{5}, t, r}\left(F_{\lambda_{5},(r, t) ; \mathbf{1}}^{\lambda_{1}, \lambda_{2}}\right)^{2}=\frac{1}{D_{\lambda_{1}} D_{\lambda_{2}}} \sum_{\lambda_{5}, t} D_{\lambda_{5}}=\frac{1}{D_{\lambda_{1}} D_{\lambda_{2}}} \sum_{\lambda_{5}} N_{\lambda_{1} \lambda_{2}}^{\lambda_{5}} D_{\lambda_{5}}=1
$$

There exists a basis such that upon complex conjugation

$$
\left(d_{\Lambda_{1} \Lambda_{2}}^{\Lambda_{5} ;(t, \bar{t})}\right)^{*}=d_{\Lambda_{1}^{*} \Lambda_{2}^{*}}^{\Lambda_{*}^{*} ;\left(t^{*} \bar{t}^{*}\right)}
$$

Choosing $d_{\Lambda^{*} \Lambda}^{\mathbf{1}}=(-1)^{s(\Lambda)}$, we have furthermore (from the locality of the 3 -point function and the above choice of $\left.F_{\lambda_{5},(r, t) ; \mathbf{1}}^{\lambda_{1}, \lambda_{2}}\right)$

$$
d_{\Lambda_{2}^{*} \Lambda_{5}}^{\Lambda_{1} ; B}=(-1)^{s\left(\Lambda_{1}\right)+s\left(\Lambda_{5}\right)} d_{\Lambda_{1}^{*} \Lambda_{2}^{*}}^{\Lambda_{*}^{*} \sigma_{123}(B)},
$$


and hence (3.3) simplifies to

$$
\sum_{\Lambda_{5}} \sum_{t, \bar{t}}\left|d_{\Lambda_{1} \Lambda_{2}}^{\Lambda_{5} ;}\right|^{2} F_{\lambda_{1} \lambda_{2}}^{\lambda_{5}} F_{\bar{\lambda}_{1} \bar{\lambda}_{2}}^{\bar{\lambda}_{5}}=1
$$

There is still a "gauge" freedom left in determining the constants $d^{A}$ : a transformation $P_{t t^{\prime}} \otimes \bar{P}_{\overline{t t^{\prime}}}$ by an unitary matrix $P_{t t^{\prime}}$ keeps invariant equation (3.3) and the chosen normalisations. 4 In the $\operatorname{sl}(2)$ case the constants $d_{(\lambda, \lambda)(\mu, \mu)}^{(\nu, \nu)}$ coincide with the relative structure constants represented by the r.h.s. of (2.23).

We shall make now, following the idea in [19, two assumptions which were justified in the $\operatorname{sl}(2)$ case [3] by the explicit solutions of the duality equations.

Consider a theory described by a modular invariant partition function of type I (2.9), and denote alternatively by $\{\lambda\}$ the representation $\mathcal{B}_{i}$ of the extended algebra, if $\lambda$ appears in its decomposition with respect to $\widehat{s l}(n)_{k}$; recall that the dimensions $\triangle_{\lambda}$ for $\lambda \in\{\lambda\}$ differ by integers. Each field operator in these cases is described by a pair of weights $(\lambda, \bar{\lambda})$ such that both $\lambda, \bar{\lambda} \in\{\lambda\}$. We shall make a frequent use of this feature, carrying out the summation over pairs of weights (like $\Lambda_{5}$ in (3.8) ) in two steps: a summation over blocks and a summation over weights within that block. We shall assume that in all nondiagonal cases associated with conformal embeddings the constants $d^{A}$ factorise according to

$$
N_{\left\{\lambda_{1}\right\},\left\{\lambda_{2}\right\}}^{\left\{\lambda_{5}\right\}} \sum_{t, \bar{t}}\left|d_{\Lambda_{1} \Lambda_{2}}^{\Lambda_{5} ; A}\right|^{2}=\widetilde{M}_{\lambda_{1} \lambda_{2}}^{\lambda_{5}} \widetilde{M}_{\bar{\lambda}_{1} \bar{\lambda}_{2}}^{\bar{\lambda}_{\bar{N}}}
$$

where $N_{\left\{\lambda_{1}\right\}\left\{\lambda_{2}\right\}}^{\left\{\lambda_{5}\right\}}$ are the Verlinde multiplicities of the extended theory, $\lambda_{i}, \bar{\lambda}_{i} \in\left\{\lambda_{i}\right\}$, and $\widetilde{M}_{\lambda_{1} \lambda_{2}}^{\lambda_{5}}$ are some real constants. They are defined only whenever $N_{\lambda_{1} \lambda_{2}}^{\lambda_{5}} \neq 0, N_{\left\{\lambda_{1}\right\}\left\{\lambda_{2}\right\}}^{\left\{\lambda_{5}\right\}} \neq 0$, and will be considered to be identically zero otherwise. The above formula, which allows to block-diagonalise (3.8), makes sense in the diagonal case as well. Indeed in that case $N_{\left\{\lambda_{1}\right\}\left\{\lambda_{2}\right\}}^{\left\{\lambda_{5}\right\}}$ coincides with the Verlinde multiplicity and (3.9) reduces to $\widetilde{M}_{\lambda_{1} \lambda_{2}}^{\lambda_{5}}=N_{\lambda_{1} \lambda_{2}}^{\lambda_{5}}$. In the nondiagonal cases related to conformal embeddings of $\widehat{s l}(n)_{k}$ into a simple affine KM algebra $\hat{g}$ at level 1 the multiplicities $N_{\left\{\lambda_{1}\right\}\left\{\lambda_{2}\right\}}^{\left\{\lambda_{5}\right\}}$ take the values 1,0 in all cases.

We insert (3.9) into (3.8) and make now the second assumption that the quantity

$$
N_{\left\{\lambda_{1}\right\}\left\{\lambda_{2}\right\}}^{\left\{\lambda_{5}\right\}} F_{\left\{\lambda_{1}\right\}\left\{\lambda_{2}\right\}}^{\left\{\lambda_{5}\right\}}:=\sum_{\lambda_{5} \in\left\{\lambda_{5}\right\}} \widetilde{M}_{\lambda_{1} \lambda_{2}}^{\lambda_{5}} F_{\lambda_{1} \lambda_{2}}^{\lambda_{5}}
$$

4 The matrices $P$ may depend on the triplet of weights, i.e., $P=P_{\lambda_{1} \lambda_{2}}^{\lambda_{5}}$. The general duality equations are covariant when transforming both $d$ and $F$. Furthermore they are invariant under the change $d_{\Lambda_{1} \Lambda_{2}}^{\Lambda_{5} ; A} \rightarrow \mu_{\Lambda_{1}} \mu_{\Lambda_{2}} \bar{\mu}_{\Lambda_{5}} d_{\Lambda_{1} \Lambda_{2}}^{\Lambda_{5} ; A},\left|\mu_{\Lambda}\right|=1$, which preserves the 2-point function normalisation. 
depends on $\lambda_{1} \in\left\{\lambda_{1}\right\}, \lambda_{2} \in\left\{\lambda_{2}\right\}$ only through the equivalence classes $\left\{\lambda_{1}\right\},\left\{\lambda_{2}\right\}$.

With these assumptions eq. (3.8) implies

$$
\sum_{\left\{\lambda_{5}\right\}} N_{\left\{\lambda_{1}\right\}\left\{\lambda_{2}\right\}}^{\left\{\lambda_{5}\right\}}\left(F_{\left\{\lambda_{1}\right\}\left\{\lambda_{2}\right\}}^{\left\{\lambda_{5}\right\}}\right)^{2}=1 .
$$

To be a function of the classes $\left\{\lambda_{1}\right\}\left\{\lambda_{2}\right\}, F_{\left\{\lambda_{1}\right\}\left\{\lambda_{2}\right\}}^{\left\{\lambda_{5}\right\}}$ has to be representable in all possible ways by the $\widetilde{M}$ 's according to 3.10). Thus given $F_{\left\{\lambda_{1}\right\}\left\{\lambda_{2}\right\}}^{\left\{\lambda_{5}\right\}}$, satisfying (3.11), we get a linear system of equations for the unknown $\widetilde{M}_{\lambda_{1} \lambda_{2}}^{\lambda_{5}}$.

The factor $F_{\left\{\lambda_{1}\right\}\left\{\lambda_{2}\right\}}^{\left\{\lambda_{5}\right\}}$ in the l.h.s. of $(3.10)$ can be interpreted as the counterpart in the extended theory of the particular fusion matrix elements in (3.3) and (3.4); accordingly we can choose

$$
F_{\left\{\lambda_{1}\right\}\left\{\lambda_{2}\right\}}^{\left\{\lambda_{5}\right\}}=\sqrt{\frac{D_{\left\{\lambda_{5}\right\}}}{D_{\left\{\lambda_{1}\right\}} D_{\left\{\lambda_{2}\right\}}}},
$$

where $D_{\{\lambda\}}=S_{\{\lambda\}\{\mathbf{1}\}} / S_{\{\mathbf{1}\}\{\mathbf{1}\}}$ is the extended quantum dimension expressed by the extended modular matrix $S_{\{\lambda\}\{\mu\}}$. The set of equations (3.11) follows from the requirement of locality of the diagonal correlators of the extended theory and (3.10) can be seen as a consistency condition for (3.11) to hold.

This kind of argumentation can be extended as discussed in the third appendix of reference [3]. Namely starting from the general system of equations implied by locality one can recover the full fusion matrix of the extended theory and convert the initial duality equations to the extended ones. Accordingly a more general factorisation assumption, replacing (3.9), can be made for the constants $d^{A}$ themselves before a summation over $A$. However the resulting set of equations for the chiral "halves" of $d^{A}$, which would replace (3.10), involves the unknown general fusion matrix elements $F_{\lambda_{5},(t, r) ; \lambda_{6},(s, u)}^{\lambda_{1}, \lambda_{2}}$. Thus, although the full extended fusion matrices are simple and in principle known (recall that the level of the extended algebra is $k=1$ ), this system cannot be effectively used to find the unknown structure constants $d$, as has been done in the rank 1 case (see Appendix $\mathrm{C}$ of [3]). Our strategy in what follows will be to study instead the simpler equations (3.10) for the matrices $\widetilde{M}$. The knowledge of the latter provides partial information about the structure constants $d$ and completely determines them whenever the corresponding Verlinde multiplicity is equal to 1.

\section{Remarks}

1) As suggested by the example $\widehat{s l}(2)_{4} \subset \widehat{s l}(3)_{1}$ (i.e., the " $D_{4}$ " case), in which there is a degeneracy of fields, we cannot expect the factorisation (3.9) to always hold in 
the "unitary basis" , i.e., for $d^{A}$ satisfying (3.6). Indeed, in that case as well as for the whole $D_{\text {even }}(\mathrm{sub})$ series $k=4 \bmod 8$, the basis for which the relative structure constants are real (recall that $\Lambda^{*}=\Lambda$ in the $\operatorname{sl}(2)$ case) excludes the appearance of more than one of the "doubled" scalar fields in any product of fields and the $d$ 's do not factorise. Complex linear combinations of these real scalar fields yields a pair of mutually conjugate fields and complex structure constants. The latter satisfy a generalisation of (3.6), which takes into account the hermitian conjugation of the two new scalar fields. Only in this basis the factorisation (3.9) takes place. Assuming that similar considerations extend to higher rank we shall use (3.10) also in cases with degeneracies of the exponents. With a slight abuse of notation we continue to use $\{\lambda\}$ for the different representations of the extended algebra containing the $\widehat{s l}(n)_{k}$ representation $\lambda$. However in all concrete examples a clear distinction will be made, adding an additional index to $\lambda$ so that no confusion arises. The same will be done when $\lambda$ appears with a nontrivial multiplicity $\operatorname{mult}_{\{\lambda\}}(\lambda)$ in a given representation $\{\lambda\}$; in expressions like the r.h.s. of 3.10 it will be assumed that the summation runs over all such exponents, i.e., $\sum_{\lambda \in\{\lambda\}}(\ldots)=\sum_{\lambda \in \mathcal{P}_{++}^{(h)}} \operatorname{mult}_{\{\lambda\}}(\lambda)(\ldots)$, thus accounting for the multiplicities. In fact in most of what follows (with the exception of Appendix A and sect. 5.4) we shall restrict ourselves to the cases with trivial multiplicities, $\operatorname{mult}_{\{\lambda\}}(\lambda)=0,1$.

2) We recall that in an appropriate basis the factorisation (3.9) holds true also in the $\operatorname{sl}(2)$ block-diagonal $D_{\text {even }}$ series - whenever the structure constants involve the fixed points of (2.2). Furthermore the remaining constants factorise similarly to (3.9), but in a weaker sense, up to a nonchiral $\delta$ - function factor in the r.h.s., due to an intrinsic $\mathbb{Z}_{2}$ grading of the 2-dimensional OPA [3]; also the extended multiplicity does not appear in the l.h.s. of (3.9). Although a block-diagonalisation of (3.3) of the kind performed above is impossible in general, (3.11) can be effectively recovered, since the (chiral) equation (3.10) is still valid. The only difference is that the extended multiplicity $N_{\left\{\lambda_{1}\right\}\left\{\lambda_{2}\right\}}^{\left\{\lambda_{5}\right\}}$ in $(3.10)$ and $(3.11)$ can take also the value 2 .

Combining (3.4) and (3.12) we rewrite $(3.10)$

$$
N_{\left\{\lambda_{1}\right\}\left\{\lambda_{2}\right\}}^{\left\{\lambda_{3}\right\}}=\sqrt{\frac{D_{\left\{\lambda_{1}\right\}}}{D_{\lambda_{1}}}} \sqrt{\frac{D_{\left\{\lambda_{2}\right\}}}{D_{\lambda_{2}}}} \sum_{\lambda_{3} \in\left\{\lambda_{3}\right\}} \widetilde{M}_{\lambda_{1} \lambda_{2}}^{\lambda_{3}} \sqrt{\frac{D_{\lambda_{3}}}{D_{\left\{\lambda_{3}\right.}}}, \quad \begin{aligned}
& \lambda_{1} \in\left\{\lambda_{1}\right\} \\
& \lambda_{2} \in\left\{\lambda_{2}\right\}
\end{aligned}
$$


This system of equations (3.13) that has been derived for the thermal fields of $W_{n}$ coset CFT can be analogously derived for the $\widehat{s l}(n)_{k}$ WZW models.

The constants $\widetilde{M}$ will soon be shown to be identical with the structure constants $M$ $(2.19 a)$ of the Pasquier algebra. Apparently the input in (3.13) is very simple - we need the fusion rules of the extended theory and the quantum dimensions of both the extended and unextended theories, all of which are known, being expressed by the modular matrices of the corresponding WZW models. The unextended fusion rules are also implicitly taken into account since $\widetilde{M}_{\lambda_{1} \lambda_{2}}^{\lambda_{3}} \equiv 0$ if $N_{\lambda_{1} \lambda_{2}}^{\lambda_{3}}=0$. In fact this simplicity of (11) limits its applicability since in general the set of independent equations in (11) is not sufficient to determine completely the constants $\widetilde{M}_{\lambda_{1} \lambda_{2}}^{\lambda_{3}}$ for all values of the couplings $\left(\lambda_{1}, \lambda_{2}, \lambda_{3}\right)$; rather we get some relations between these constants. Yet there are some examples, to be discussed in the next section, in which the above data provide a full solution.

As suggested by the $D_{\text {even }} \operatorname{sl}(2)$ case, the formula (3.13) might actually apply to all type I theories, though related in a more subtle way to the duality equations of the CFT, since some substitute of $(3.9)$ is required. We check and confirm this conjecture in Appendix B for the block-diagonal sl(3) modular invariants of the $D$ series, comparing with the results in [20], [17].

\section{Examples of solutions of (3.13).}

Let us illustrate the previous considerations on the case of non trivial $\widehat{s l}(n)_{k}$ theories obtained by conformal embeddings into a simple affine algebra $\hat{g}$. The latter have been classified [21], see also [15, 16]. The algebra $\widehat{s l}(n)$ may be conformally embedded into a few exceptional algebras, necessarily of level 1, and there are (restricting to embeddings into simple algebras) four infinite series of conformal embeddings

$$
\begin{aligned}
\widehat{s l}(n)_{n-2} & \subset \widehat{s l}\left(\frac{n(n-1)}{2}\right)_{1}, \quad n \geq 4, \\
\widehat{s l}(n)_{n+2} & \subset \widehat{s l}\left(\frac{n(n+1)}{2}\right)_{1}, \\
\widehat{s l}(2 n)_{2 n} & \subset \widehat{s o}\left(4 n^{2}-1\right)_{1}, \quad n \geq 2, \\
\widehat{s l}(2 n+1)_{2 n+1} & \subset \widehat{s o}(4 n(n+1))_{1} .
\end{aligned}
$$


We shall look for solutions $\widetilde{M}_{\lambda_{1} \lambda_{2}}^{\lambda_{3}}$ of the system of equations (3.13), which satisfy the symmetry relations

$$
\begin{gathered}
\widetilde{M}_{\lambda_{1} \lambda_{2}}^{\lambda_{3}}=\widetilde{M}_{\lambda_{2} \lambda_{1}}^{\lambda_{3}}=\widetilde{M}_{\lambda_{1}^{*} \lambda_{2}^{*}}^{\lambda_{*}^{*}}=\widetilde{M}_{\lambda_{1} \lambda_{3}^{*}}^{\lambda_{2}^{*}}, \\
\widetilde{M}_{\lambda \lambda^{*}}^{\rho}=1=1=\widetilde{M}_{\lambda \rho}^{\lambda},
\end{gathered}
$$

in agreement with the symmetry of the structure constants $d$ and (3.2). All solutions that we will find are consistent with the symmetry under the $\mathbb{Z}_{n}$ automorphism (2.2)

$$
\widetilde{M}_{\lambda_{1} \sigma\left(\lambda_{2}\right)}^{\sigma\left(\lambda_{3}\right)}=\widetilde{M}_{\lambda_{1} \lambda_{2}}^{\lambda_{3}}
$$

Recall that there is a general and explicit formula for the quantum dimensions in the $\widehat{s l}(n)_{k}$ affine algebra in terms of scalar products with positive roots, $D_{\lambda}=$ $\prod_{\alpha>0} \frac{\sin ((\lambda, \alpha) \pi /(k+n))}{\sin ((\rho, \alpha) \pi /(k+n))}$. They satisfy various properties like $D_{\lambda}=D_{\lambda^{*}}=D_{\sigma(\lambda)}$ and $D_{\lambda}>0$ for integrable representations. The case $\operatorname{sl}(2)$ was considered in [3]. The system (3.13) is sufficient to recover all $\widetilde{M} \equiv M$ for the block-diagonal invariants labelled $D_{4}$ $(k=4)$ and $E_{6}(k=10)$, while the case $E_{8}(k=28)$ requires considering a system of equations larger than (3.13), as discussed above.

Let turn now to the case $\operatorname{sl}(3)$. There are four values of the level, $k=3,5,9,21$, for which there exist modular invariants originating from some diagonal modular invariant of the embedding algebra. The $\widehat{s l}(3)$ modular invariant for $k=3$ corresponds to the embedding $\widehat{s l}(3)_{3} \subset \widehat{s o}(8)_{1}$, and it is also one of the invariants of the $\mathcal{D}$ series for $k=0$ $\bmod 3,22$

$$
Z_{\widehat{s l}(3)_{3}}=\left|\chi_{11}+\chi_{41}+\chi_{14}\right|^{2}+3\left|\chi_{22}^{2}\right|
$$

The integrable representations of $\widehat{s o}(8)_{1}$, are given by the identity $(1,1,1,1)$ and $(2,1,1,1)$, $(1,1,2,1),(1,1,1,2)$ (with standard notations for the $s o(n)$ weights). All have quantum dimensions equal to 1 and the charge conjugation is trivial. Their fusion algebra is of type $\mathcal{D}_{2} \cong \mathbb{Z}_{2} \times \mathbb{Z}_{2}$

$$
\begin{aligned}
& w_{i} \times w_{i}=\mathbf{1}, \quad i=1,2,3, \\
& w_{i} \times w_{j}=w_{k}, \quad i \neq j \neq k \neq i .
\end{aligned}
$$

The three identical representations $(2,2)$ of $\widehat{s l}(3)_{3}$ are distinguished naturally by assigning to each of them the index $i$ inherited from the $\widehat{s o}(8)_{1}$ counterpart. The only nontrivial 
$\widehat{s l}(3)_{3}$ quantum dimension is $D_{2,2}=3$. The system reduces, after taking into account $(4.2)$, to

$$
\begin{aligned}
& 1=\widetilde{M}_{(2,2)_{i}(4,1)}^{(2,2)_{i}}, \quad 1=\widetilde{M}_{(4,1)(4,1)}^{(1,4)}, \\
& 1=\widetilde{M}_{(2,2)_{i}(2,2)_{j}}^{(2,2)_{k}} \frac{1}{\sqrt{D_{(2,2)}}}, \quad \text { for } \quad i \neq j \neq k \neq i \\
& 1=\left(\widetilde{M}_{(2,2)_{i}(2,2)_{i}}^{(1,1)}+\widetilde{M}_{(2,2)_{i}(2,2)_{i}}^{(4,1)}+\widetilde{M}_{(2,2)_{i}(2,2)_{i}}^{(1,4)}\right) \frac{1}{D_{(2,2)}},
\end{aligned}
$$

which implies using (4.2) again

$$
\begin{aligned}
& \widetilde{M}_{(2,2)_{i}(2,2)_{j}}^{(2,2)_{k}}=\sqrt{3}, \text { for } i \neq j \neq k \neq i \\
& \widetilde{M}_{(2,2)_{i}(2,2)_{i}}^{(1,1)}=\widetilde{M}_{(2,2)_{i}(2,2)_{i}}^{(4,1)}=\widetilde{M}_{(2,2)_{i}(2,2)_{i}}^{(1,4)}=1 .
\end{aligned}
$$

The last two equalities in (4.6) incorporate the $\widehat{s l}(3)_{3}$ fusion rule

$$
(2,2) \otimes(2,2)=(1,1) \oplus(1,4) \oplus(4,1) \oplus 2(2,2)
$$

in a way respecting the fusion rule (4.5) of the corresponding extended representations. The remaining matrix elements are recovered from (4.2); (4.3) is indeed fulfilled.

In this and in all examples to follow the degeneracy of fields with the same $\operatorname{sl}(n)$ weight is resolved by the correspondence to different representations of the extended algebra. In all these cases the symmetry relations have to be reformulated for the pairs of indices, taking into account also the conjugation properties (trivial in the above example) of the extended fusion multiplicities.

To the modular invariant (4.4) has been assigned in [20] [17] the graph $\mathcal{D}^{(6)}$ of Fig. 1. For an appropriate choice of the eigenvectors $\psi$, the $M$ 's computed according to (2.19a) coincide with the $\widetilde{M}$ just determined. This coincidence can be also checked on the example $k=5$. The WZW exceptional modular invariant described by the conformal embedding $\widehat{s l}(3)_{5} \subset \widehat{s l}(6)_{1}$ reads

$$
Z_{\widehat{s l}(3)_{5}}=\left|\chi_{11}+\chi_{33}\right|^{2}+\left|\chi_{13}+\chi_{43}\right|^{2}+\left|\chi_{23}+\chi_{61}\right|^{2}+\left|\chi_{41}+\chi_{14}\right|^{2}+\left|\chi_{32}+\chi_{16}\right|^{2}+\left|\chi_{31}+\chi_{34}\right|^{2}
$$

The integrable representations of $\widehat{s l}(n)_{1}$ consist of the identity $\rho=\sum_{i=1}^{n-1} \hat{\Lambda}_{i}$, and the fundamental representations $\hat{\Lambda}_{1}+\rho=\sigma(\rho), \cdots, \hat{\Lambda}_{n-1}+\rho=\sigma^{n-1}(\rho)$. They close on a $\mathbb{Z}_{n}$ type of fusion algebra, with $\lambda_{i}:=\hat{\Lambda}_{i}+\rho$, being identified with $\sigma^{i}$, and have quantum dimensions equal to 1 . In our example these representations correspond to the blocks in 
the above order, i.e., $\chi_{13}+\chi_{43} \rightarrow \sigma, \chi_{23}+\chi_{61} \rightarrow \sigma^{2}$, etc. The quantum dimensions of the relevant representations of $\widehat{s l}(3)_{5}$ read

$$
\begin{aligned}
& D_{3,3}=D_{3,2}=(1+\sqrt{2})^{2}=3+2 \sqrt{2} \\
& D_{1,3}=D_{1,4}=D_{3,4}=2+\sqrt{2} \\
& D_{1,1}=D_{1,6}=1
\end{aligned}
$$

Now we can solve the system (3.13), imposing the restrictions of the $\mathbb{Z}_{n}$ fusion algebra of the classes on the corresponding representatives, while the sum in the r.h.s. goes over representations $\lambda_{3}$ of $\widehat{s l}(3)_{5}, \lambda_{3} \in\left\{\lambda_{3}\right\}$, such that the Verlinde multiplicities $N_{\lambda_{1} \lambda_{2}}^{\lambda_{3}}$ are nonzero. Taking into account the symmetry relations (4.2), (4.3), the result for the independent nonzero matrix elements is

$$
\begin{aligned}
& \widetilde{M}_{(3,3)(3,3)}^{(3,3)}=\sqrt{D_{3,3}}-\frac{1}{\sqrt{D_{3,3}}}=2 \\
& \widetilde{M}_{(3,1)(4,3)}^{(3,3)}=\frac{D_{(1,3)}}{\sqrt{D_{3,3}}=\sqrt{2}} \\
& \widetilde{M}_{(3,3)(3,1)}^{(3,1)}=1=\widetilde{M}_{(3,3)(3,2)}^{(1,6)}=\widetilde{M}_{(3,3)(4,3)}^{(4,3)}=\widetilde{M}_{(6,1)(3,1)}^{(4,3)}=\widetilde{M}_{(6,1)(1,3)}^{(4,1)}
\end{aligned}
$$

which is in agreement with the values for the corresponding matrices $M$ attached to the graph $\mathcal{E}^{(8)}$ introduced in [17] (see Fig. 1).
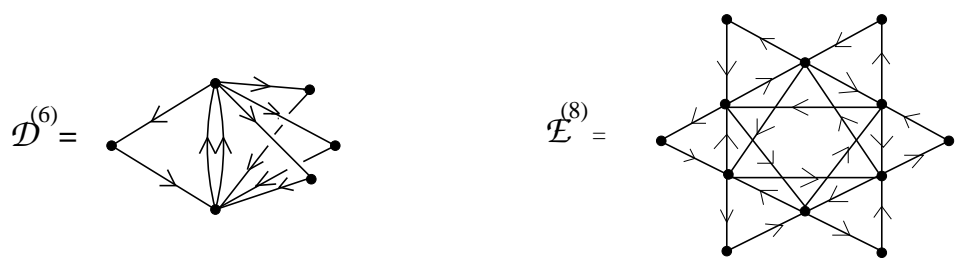

Fig. 1: The oriented graphs $\mathcal{D}^{(6)}$ and $\mathcal{E}^{(8)}$ of matrix $G_{1}$ for the conformal embeddings of $\widehat{s l}(3)_{3} \subset \widehat{s o}(8)_{1}$ and $\widehat{s l}(3)_{5} \subset \widehat{s l}(6)_{1}$

The system (3.13) can be analysed also in the remaining two exceptional cases $k=$ 9,21 , corresponding to the embeddings of $\widehat{s l}(3)$ into level $1 \widehat{E_{6}}$ and $\widehat{E_{7}}$ respectively. Again all quantum dimensions of the representations of the extended algebras are equal to 1 , while their fusion algebras are of type $\mathbb{Z}_{3}$ and $\mathbb{Z}_{2}$ respectively. However the equations in these cases are not sufficient to determine all matrix elements. Nevertheless they impose a set of restrictions, which can be checked to be consistent with the explicit results of [17]. As a side remark, we note that in the case $k=9$ some of the squares of the $M$ matrix 
elements are not given by rational numbers. Recall that they are rational in the $s l(2)$ case as a general property [3], [24].

Based on the results in the $\operatorname{sl}(2)$ and $\operatorname{sl}(3)$ cases we now make the conjecture that the matrices $M$ and $\widetilde{M}$ introduced in (2.19a) and (3.9) are identical in general. Hence we look for new solutions of (3.13), to be used to reconstruct the corresponding graphs as explained in sect. 1.

The first two non trivial cases of the first series in (4.1) are $\widehat{s l}(4)_{2} \subset \widehat{s l}(6)_{1}$ and $\widehat{s l}(5)_{3} \subset \widehat{s l}(10)_{1}$, for which the modular invariant partition functions read respectively

$$
\begin{aligned}
Z_{\widehat{s l}(4)_{2}} & =\left|\chi_{111}+\chi_{131}\right|^{2}+2\left|\chi_{121}\right|^{2}+2\left|\chi_{212}\right|^{2}+\left|\chi_{113}+\chi_{311}\right|^{2} \\
& Z_{\widehat{s}(5)_{3}}=\left|\chi_{1111}+\chi_{1221}\right|^{2}+\left|\chi_{3121}+\chi_{1213}\right|^{2} \\
+ & \left\{\left|\chi_{1211}+\chi_{2131}\right|^{2}+\left|\chi_{1411}+\chi_{1212}\right|^{2}+\left|\chi_{1311}+\chi_{2113}\right|^{2}+\left|\chi_{4111}+\chi_{2122}\right|^{2}+\text { c.c. }\right\}
\end{aligned}
$$

In the case (4.11a), the successive blocks correspond respectively to the weights $\lambda_{0}=$ $\rho, \lambda_{1}$ and $\lambda_{5}, \lambda_{2}$ and $\lambda_{4}$, and $\lambda_{3}$ of $\widehat{s l}(6)_{1}$, where we are making use of the notation $\lambda_{i}=\rho+\hat{\Lambda}_{i}$ introduced above for the fundamental weights shifted by $\rho$. In the case (1.11b) likewise, the blocks correspond to $\lambda_{0}, \lambda_{5}$, followed by $\lambda_{1}, \lambda_{2}, \lambda_{3}, \lambda_{4}$ and their conjugate. For each of these cases, the system (3.13) fully determines the $\widetilde{M}$ 's. In the case of $\widehat{s l}(4)_{2}$, the degeneracy of, say, the two representations $\lambda=(1,2,1)$ is lifted by the assignment of two opposite $\mathbb{Z}_{6}$ charges equal \pm 1 that they inherit from the "parent" representations $(2,1,1,1,1)$ and $(1,1,1,1,2)$ in $\widehat{s l}(6)_{1}$. The symmetry relations (4.2) hold with, e.g., $\left((1,2,1)_{ \pm}\right)^{*}=(1,2,1)_{\mp}$, etc. We thus obtain for the matrix $\widetilde{M}_{(121)_{+}}$in the basis $(111),(131),(121)_{+},(212)_{+},(311),(113),(212)_{-},(121)_{-}$

$$
\widetilde{M}_{(121)_{+}}=\left(\begin{array}{cccccccc} 
& & & & & & & \\
& 1 & & & & & \\
& & \sqrt{2} & & & & \\
& & & 1 & 1 & & \\
& & & & & 1 & \\
& & & & & 1 & \\
& & & & & & & \sqrt{2} \\
1 & 1 & & & & & &
\end{array}\right) .
$$

Now assuming that $\widetilde{M}=M$ and diagonalizing it as in (2.19a), we may determine the $\psi$ and construct the matrices $N$. As anticipated their entries are all non negative integers and one of them, arbitrarily denoted $N_{7}$, turns out to have the desired spectrum to qualify 

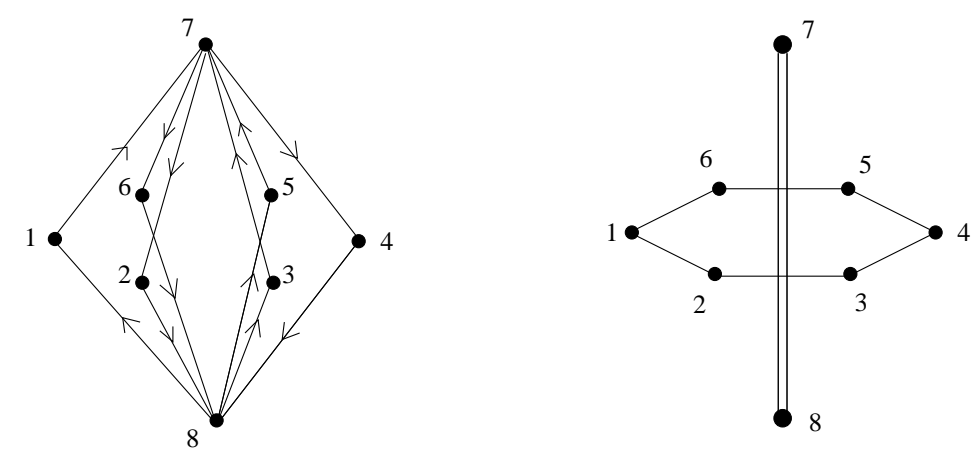

Fig. 2: The graphs of $G_{1}$ and $G_{2}$ corresponding to the conformal embedding $\widehat{s l}(4)_{2} \subset \widehat{s l}(6)_{1}$. The involution $a \rightarrow a^{\vee}$ acts as the reflection in the line 1-4 if the graph is regarded as two dimensional.
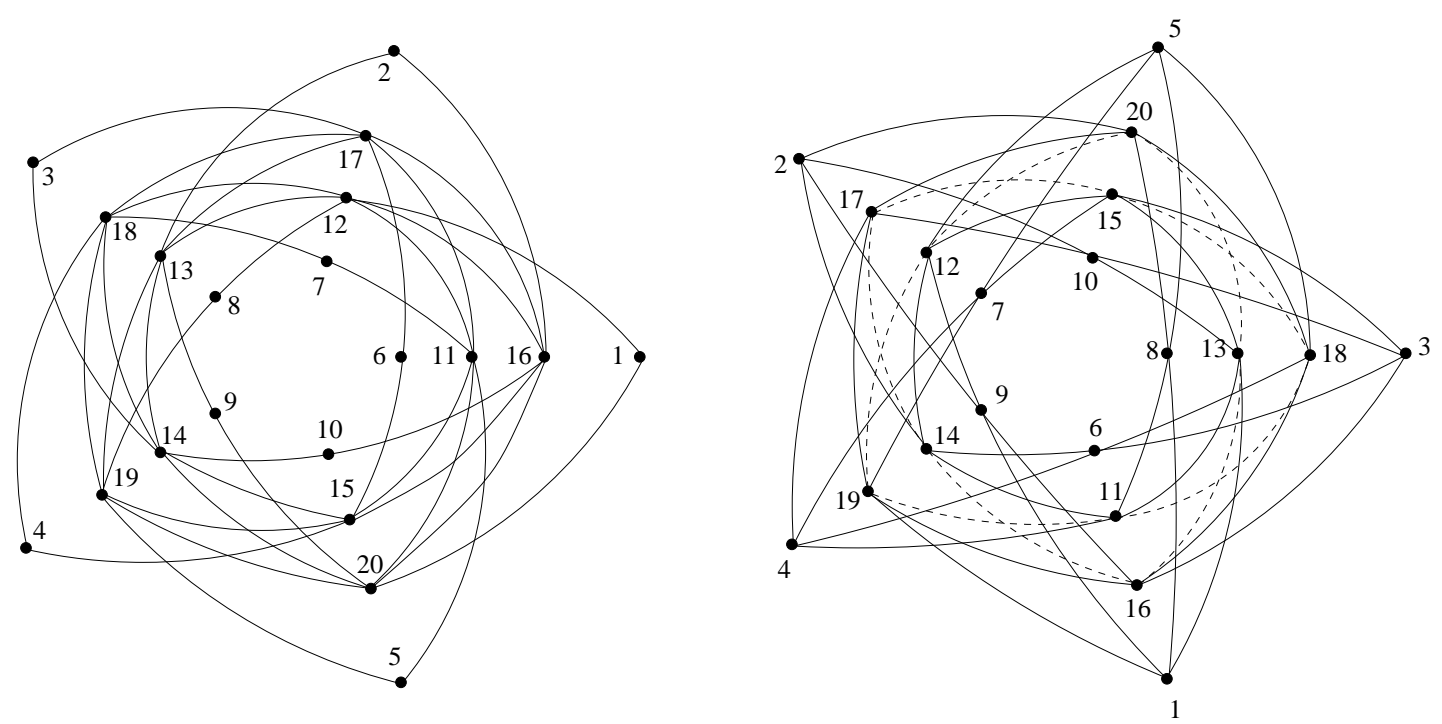

Fig. 3: The graphs of $G_{1}$ and $G_{2}$ corresponding to the conformal embedding $\widehat{s l}(5)_{3} \subset \widehat{s l}(10)_{1}$; see the text for the conventions on the 5 -ality of the vertices and the ensuing orientations of edges; for the graph of $G_{2}$ a certain reshuffling of the radial directions has been done for more readability and broken lines indicate double edges. The involution $a \rightarrow a^{\vee}$ is the reflection in the line 1-6 for $1 \leq a \leq 10$ whereas $11^{\vee}=16$ and $a^{\vee}=32-a, 12 \leq a \leq 20$.

as $G_{1}$ : its eigenvalues are of the form (2.16) with $\lambda$ taking all the values that appear in the diagonal terms of (4.11a). The graph is depicted in Fig. 2 together with the graph of the matrix $G_{2}=N_{2}+N_{6}$ also identified by its spectrum; finally $G_{3}=G_{1}^{t}=N_{8}$. The same procedure with the case of $s l(5)_{3}$ produces the graphs of Fig. 3. In fig. 3.a, is represented the graph of matrix $G_{1}=N_{12}$, with a certain labelling of the vertices: this labelling has been chosen in such a way that a vertex marked $\ell$ had a "5-ality" equal to $\ell-1 \bmod 5$. In fig 3.b, is represented the graph of $G_{2}=N_{8}+N_{18}$. The matrices $G_{3}=G_{2}^{t}, G_{4}=G_{1}^{t}$ 
are also found among the $N$ matrices or their combinations: $G_{3}=N_{9}+N_{19}, G_{4}=N_{20}$. The arrows of these graphs have been omitted as redundant since they are dictated by rule $(2.13)$.

\section{Dual pairs of $C$ - algebras - properties and applications.}

In this section we transform (3.13) into a system of equations for the eigenvectors $\psi_{a}$ of the $M$ - matrices and in particular we obtain for a subset of them explicit analytic expressions valid for arbitrary $\operatorname{sl}(n)$. For this purpose we need some properties of the $C$ ("character") - algebras [25], so let us briefly review the basic notions and some theorems involved.

\subsection{A short review of the theory of $C$-algebras}

A $C$ - algebra $\mathcal{U}$ is an associative commutative algebra over $\mathbb{C}$, with a basis $x_{1}, x_{2}, \ldots, x_{d}$, real structure constants,

$$
x_{a} x_{b}=\sum_{c} p_{a b}^{c} x_{c}, \quad p_{a b}^{c} \in \mathbb{R}
$$

an identity $x_{1}$, (to be also denoted by $x_{1}$ ), hence $p_{1 b}^{a}=\delta_{a b}$, and an involution $a \rightarrow a^{\vee}$, which extends to an algebra isomorphism $x_{a} \rightarrow\left(x_{a}\right)^{\vee}:=x_{a^{\vee}}-$ hence $p_{a b}^{c}=p_{a^{\vee} b^{\vee}}^{c^{\vee}}$, and such that

$$
p_{a b}^{1}=\delta_{a b \vee} k_{a}, \quad k_{a}>0 \quad \forall a, b .
$$

It is furthermore assumed that the mapping $x_{a} \rightarrow k_{a}$ is a linear representation of $\mathcal{U}$. It follows that $\mathbf{1}^{\vee}=\mathbf{1}, \quad k_{\mathbf{1}}=1, \quad k_{a^{\vee}}=k_{a}$, and $k_{c} p_{a b}^{c}=k_{b} p_{a^{\vee} c}^{b}=k_{b} p_{a c^{\vee}}^{b^{\vee}}$. The algebra is realised isomorphically by the matrix commutative algebra spanned by $\left(p_{a}\right)_{b}^{c}, a=1, . ., d$.

These axioms imply furthermore that the matrices $\left(B_{a}\right)_{b}^{c}=\frac{\sqrt{k_{c}}}{\sqrt{k_{b}}}\left(p_{a}\right)_{b}^{c}$ are normal (since $B_{a}$ commute and ${ }^{t} B_{a}=B_{a \vee}$ ) and hence can be diagonalised simultaneously by an unitary matrix $U$, i.e.,

$$
\frac{\sqrt{k_{c}}}{\sqrt{k_{b}}} p_{a b}^{c}=\sum_{i=1}^{d} U_{b i} p_{a}(i) U_{c i}{ }^{*}
$$

where the eigenvalues may be written $p_{a}(i)=\frac{U_{a i}}{U_{1 i}} \sqrt{k_{a}} ; \quad$ one may choose $p_{a}(1)=k_{a}$, and the complex conjugate of $U_{a i}$ satisfies $U_{a i}{ }^{*}=U_{a \vee}$.

The $C$ - algebra $\mathcal{U}$ has exactly $d$ linear representations $\triangle_{i}$, given by $\triangle_{i}: x_{a} \rightarrow p_{a}(i)$. 
There is a natural notion of a dual $C$ - algebra $\hat{\mathcal{U}}$, as the set of all linear mappings of $\mathcal{U}$ into $\mathbb{C}$ with the point-wise multiplication. It has structure constants $\hat{p}_{i k}^{j}$ satisfying

$$
\frac{\sqrt{\hat{k}_{k}}}{\sqrt{\hat{k}_{j}}} \hat{p}_{i j}^{k}=\sum_{a=0}^{d} U_{a j} \hat{p}_{i}(a) U_{a k}^{*},
$$

where the eigenvalues are $\hat{p}_{i}(a)=\frac{U_{a i}}{U_{a 1}} \sqrt{\hat{k}_{i}}, \quad \hat{p}_{i}(1)=\hat{k}_{i}$, and $U_{a i}{ }^{*}=U_{a i}$. The dual of $\hat{\mathcal{U}}$ is $\mathcal{U}$.

The unitary matrix $U$, which diagonalises the structure constants of the pair of algebras $\mathcal{U}$ and $\hat{\mathcal{U}}$ according to (5.3), (5.4) is in general nonsymmetric. Whenever $U$ can be chosen symmetric the algebra $\mathcal{U}$ is selfdual.

Comparing with (2.19a), (2.19b), it is clear that we can look at the pair of algebras spanned by the $N$ and $M$ - matrices as matrix realisations of a dual pair of $C$ - algebras $\mathcal{U}$ and $\hat{\mathcal{U}}$ with

$$
\begin{gathered}
\sqrt{k_{a}}=\frac{\psi_{a}^{(\rho)}}{\psi_{\mathbf{1}}^{(\rho)}}, \quad \sqrt{\hat{k}_{\lambda}}=\frac{\psi_{\mathbf{1}}^{(\lambda)}}{\psi_{\mathbf{1}}^{(\rho)}}, \\
N_{a b}^{c}=\frac{\sqrt{k_{c}}}{\sqrt{k_{a}} \sqrt{k_{b}}} p_{a b}^{c}, \quad M_{\lambda \mu}^{\gamma}=\frac{\sqrt{\hat{k}_{\gamma}}}{\sqrt{\hat{k}_{\lambda}} \sqrt{\hat{k}_{\mu}}} \hat{p}_{\lambda \mu}^{\gamma} .
\end{gathered}
$$

One introduces in an obvious way the notion of a $C$-subalgebra of the $C$-algebra $\mathcal{U}$ as the $C$-algebra $\mathcal{U}_{T}$ with basis $\left\{x_{a}, a \in T\right\}$, where $T$ is a subset of $\{1,2, . ., d\} . \mathcal{U}_{T}$ is a proper $C$-subalgebra if $1<|T|<d$.

From now on we shall assume that both $\mathcal{U}$ and $\hat{\mathcal{U}}$ have nonnegative structure constants. One can prove that under this assumption $\mathcal{U}$ has a proper $C$-subalgebra iff its dual $\hat{\mathcal{U}}$ has a proper $C$-subalgebra. (Recall that the nonnegativity of the $M$ and $N$ matrices was established for all known examples of type I theories and assumed for all general theories of this type.) Furthermore if $\mathcal{U}$ has a proper $C$-subalgebra $\mathcal{U}_{T}$ one can define a factor $C$-subalgebra, denoted $\mathcal{U} / \mathcal{U}_{T}$, by splitting the set $\{1,2, . ., d\}$ into equivalence classes $T_{1} \equiv T, T_{2}, \ldots, T_{t}$. Namely $a \sim c$ iff $\exists \beta \in T$, such that $p_{a \beta}^{c} \neq 0$. Then the elements $X_{i}:=\frac{1}{\sum_{a \in T} k_{a}} \sum_{b \in T_{i}} x_{b}, i=1,2, \ldots, t$, with the multiplication inherited from $\mathcal{U}$, provide a basis for $\mathcal{U} / \mathcal{U}_{T}$. The parameter $k_{T_{i}}$ is given by $k_{T_{i}}=\sum_{a \in T_{i}} k_{a} / \sum_{a \in T} k_{a}$. A theorem in [25] states that the dual of $\mathcal{U} / \mathcal{U}_{T}$ is isomorphic to a $C$-subalgebra of $\hat{\mathcal{U}}$, obtained by the same procedure starting from a subset $\hat{T}$ of the dual basis and thus denoted $\hat{\mathcal{U}}_{\hat{T}}$. The classes $T_{i}$ can be enumerated by the elements of the subset $\hat{T} \ni i$. Vice versa, there is one to one correspondence between the elements of $T$ and the equivalence classes $\hat{T}_{a}, a=1,2, \ldots,|T|, \hat{T}_{1} \equiv \hat{T}$, of $\hat{\mathcal{U}}$, and $\hat{\mathcal{U}} / \hat{\mathcal{U}}_{\hat{T}} \cong \widehat{\mathcal{U}_{T}}$. 


\subsection{Further analysis of Eq. (3.13)}

Let us now return to the set of equations (3.13) assuming that all its solutions for the $M=\widetilde{M}$ are nonnegative. We multiply both sides with

$$
\chi_{\left\{\lambda_{3}\right\}}(\{\gamma\}):=\frac{S_{\left\{\lambda_{3}\right\}\{\gamma\}}}{S_{\{1\}\{\gamma\}}}
$$

where $S_{\{\lambda\}\{\gamma\}}$ is the modular matrix of the extended theory. Then we sum over the classes, using that $\chi_{\{\lambda\}}(\{\gamma\})$ provide for any $\{\gamma\}$ a 1- dimensional representation of the extended fusion algebra. We thus obtain

$$
\left(\chi_{\left\{\lambda_{1}\right\}}(\{\gamma\}) \sqrt{\frac{D_{\lambda_{1}}}{D_{\left\{\lambda_{1}\right\}}}}\right)\left(\chi_{\left\{\lambda_{2}\right\}}(\{\gamma\}) \sqrt{\frac{D_{\lambda_{2}}}{D_{\left\{\lambda_{2}\right\}}}}\right)=\sum_{\lambda_{3}} M_{\lambda_{1} \lambda_{2}}^{\lambda_{3}}\left(\chi_{\left\{\lambda_{3}\right\}}(\{\gamma\}) \sqrt{\frac{D_{\lambda_{3}}}{D_{\left\{\lambda_{3}\right\}}}}\right) .
$$

It follows that we can interpret $\chi_{\{\lambda\}}(\{\gamma\}) \sqrt{\frac{D_{\lambda}}{D_{\{\lambda\}}}}$ as some of the 1 - dimensional representations of the $M$ algebra, given in general by $\frac{\psi_{c}^{(\lambda)}}{\psi_{c}^{(1)}}, c \in \mathcal{V}$. Thus identifying each class $\{\gamma\}$ with a vertex $c$, such that $\{\mathbf{1}\}$ corresponds to the identity vertex element $\mathbf{1} \in \mathcal{V}$, and $\{\gamma\}^{*}=c^{\vee}$, we select a subset $T$ of the vertices for which (5.6) provides explicit expressions for the $M$ - eigenvalues

$$
\frac{\psi_{c}^{(\lambda)}}{\psi_{c}^{(\mathbf{1})}}:=\chi_{\{\lambda\}}(\{\gamma\}) \sqrt{\frac{D_{\lambda}}{D_{\{\lambda\}}}}=\frac{S_{\{\gamma\}\{\lambda\}}}{S_{\{\gamma\}\{\mathbf{1}\}}} \sqrt{\frac{D_{\lambda}}{D_{\{\lambda\}}}}, \quad \forall \lambda \in \operatorname{Exp}, \quad\{\gamma\} \equiv c \in T,
$$

and in particular

$$
\frac{\psi_{\mathbf{1}}^{(\lambda)}}{\psi_{\mathbf{1}}^{(\mathbf{1})}}=\chi_{\{\lambda\}}(\{\mathbf{1}\}) \sqrt{\frac{D_{\lambda}}{D_{\{\lambda\}}}}=\frac{S_{\{\mathbf{1}\}\{\lambda\}}}{S_{\{\mathbf{1}\}\{\mathbf{1}\}}} \sqrt{\frac{D_{\lambda}}{D_{\{\lambda\}}}}=\sqrt{D_{\lambda} D_{\{\lambda\}}} .
$$

so that for any exponent $\lambda$, we have $\sqrt{\hat{k}_{\lambda}}=\frac{\psi_{1}^{(\lambda)}}{\psi_{1}^{(1)}}=\sqrt{D_{\lambda} D_{\{\lambda\}}}$.

Clearly the l.h.s. of (5.7) is constant when $\lambda$ varies within a given class $\{\lambda\}$ and hence we can rewrite (5.7) more symmetrically dividing by $\sqrt{D_{\{\lambda\}}}$ and summing over $\lambda \in\{\lambda\}$

$$
\chi_{\{\lambda\}}(c)=\frac{\sqrt{D_{\{\lambda\}}}}{|\{\lambda\}|} \sum_{\lambda \in\{\lambda\}} \frac{\chi_{\lambda}(c)}{\sqrt{D_{\lambda}}}, \quad c \in T
$$

where $\chi_{\lambda}(c)$ stay for the eigenvalues in the l.h.s. of $(5.7)$ and $|\{\lambda\}|=\sum_{\lambda \in \mathcal{P}_{++}} \operatorname{mult}_{\{\lambda\}}(\lambda)$. 
Using the unitarity relations (2.18a) and the fact that the extended modular matrix elements $S_{\{1\}\{\lambda\}}$ are expressed through elements of the modular matrix $S_{\lambda \mu}$ of $\widehat{s l}(n)_{k}$, i.e., $S_{\{1\}\{\lambda\}}=\sum_{\lambda \in\{\lambda\}} S_{1 \lambda}$, we obtain from (5.7)

$$
\left(\psi_{c}^{(\mathbf{1})}\right)^{2}=S_{\mathbf{1 1}} S_{\{\mathbf{1}\}\{\mathbf{1}\}} D_{\{\gamma\}}^{2}=\frac{\left(S_{\{\gamma\}\{\mathbf{1}\}}\right)^{2}}{\sum_{\alpha \in\{\mathbf{1}\}} D_{\alpha}}=\left(\psi_{\mathbf{1}}^{(\mathbf{1})}\right)^{2} D_{\{\gamma\}}^{2}, \quad c \equiv\{\gamma\} \in T .
$$

Recall that (cf. (2.18b) ) $\psi_{c}^{(\mathbf{1})}$ are real in agreement with the reality of $S_{\{\mathbf{1}\}\{\lambda\}}$ and we can furthermore choose them positive. We can thus identify all the $T$-components of the Perron-Frobenius eigenvector of the $N$ algebra

$$
\sqrt{k_{a}}=\frac{\psi_{a}^{(\mathbf{1})}}{\psi_{\mathbf{1}}^{(\mathbf{1})}}=D_{\{\alpha\}}, \quad \forall a \in T .
$$

Inserting (5.9) back into (5.7) we obtain an explicit expression for the subset of the eigenvectors $\left\{\psi_{c}, c \in T\right\}$ of the $M$ matrices

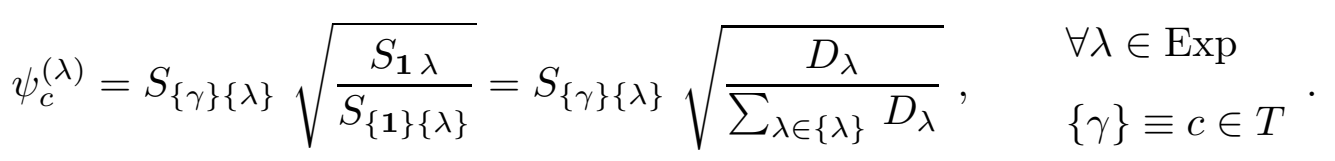

The formula (5.11) is the main result in this section. In particular it determines explicitly the Perron - Frobenius vector (of the $M$ algebra)

$$
\psi_{1}^{(\lambda)}=\sqrt{S_{1 \lambda} S_{\{1\}\{\lambda\}}}, \quad \lambda \in\{\lambda\} .
$$

Both $S_{1 \lambda}$ and $S_{\{1\}\{\lambda\}}$ are real, positive - and thus taking the positive root in (5.11), all components of $\psi_{1}$ are indeed positive. The formula (5.11) furthermore implies

$$
\frac{\psi_{c}^{(\lambda)}}{\psi_{\mathbf{1}}^{(\lambda)}}=\frac{S_{\{\gamma\}\{\lambda\}}}{S_{\{\mathbf{1}\}\{\lambda\}}}=\chi_{\{\gamma\}}(\{\lambda\}), \quad c \equiv\{\gamma\} \in T, \quad \lambda \in\{\lambda\},
$$

(i.e., the eigenvalues of $N_{c}, c \in T$, are constant within a class $\{\lambda\}$ ) and hence

$$
\begin{gathered}
\frac{\psi_{c}^{(\lambda)}}{\sqrt{\sum_{a \in T}\left|\psi_{a}^{(\lambda)}\right|^{2}}}=S_{\{\gamma\}\{\lambda\}}, \quad c \equiv\{\gamma\} \in T, \quad \lambda \in\{\lambda\}, \\
\sum_{\lambda \in\{\lambda\}} \sum_{a \in T}\left|\psi_{a}^{(\lambda)}\right|^{2}=1,
\end{gathered}
$$


since $\sum_{a \in T}\left|\psi_{a}^{(\lambda)}\right|^{2}=S_{\mathbf{1} \lambda} / S_{\{\mathbf{1}\}\{\lambda\}}$. (Note also $\sum_{\lambda \in\{\lambda\}}\left|\psi_{c}^{(\lambda)}\right|^{2}=\left|S_{\{\gamma\}\{\lambda\}}\right|^{2}$ for $c \equiv\{\gamma\} \in$ $T$, so that $\sum_{\lambda \in \operatorname{Exp}}\left|\psi_{c}^{(\lambda)}\right|^{2}=1$, in agreement with (2.18a).)

The symmetry of the extended modular matrix $S_{\{\gamma\}\{\lambda\}}=S_{\{\lambda\}\{\gamma\}}$ leads to relations for the $\psi$ 's, which, using (5.11), read

$$
\sqrt{\frac{D_{\{\beta\}}}{D_{\beta}}} \psi_{a}^{(\beta)}=\sqrt{\frac{D_{\{\alpha\}}}{D_{\alpha}}} \psi_{b}^{(\alpha)}, \quad a, b \in T, a \equiv\{\alpha\}, b \equiv\{\beta\},
$$

i.e., the ratio of quantum dimensions "deforms" the nondiagonal matrix elements $\psi_{a}^{(\beta)}$, $a, b \in T$. If each class contains only one element, i.e., $T$ coincides with $\mathcal{V}$, and $D_{\{\lambda\}} \equiv D_{\lambda}$, the symmetry of the modular matrix $S_{\lambda \mu}$ is recovered.

Combined with (2.196), equations (5.13), (5.14), (5.15) imply that the matrix elements $\left(N_{a}\right)_{b}^{c}, b, c \in T$, of the subset of $N$ matrices, $\left\{N_{a}, a \in T\right\}$ coincide with the extended Verlinde multiplicities and hence are nonnegative integers. One can show that $\left(N_{a}\right)_{b}^{c}=0$ for $a, b \in T, c \notin T$, because all $N$ matrix elements are assumed nonnegative and $k_{a}$ are strictly positive. (Hint: Use also that $k_{a}, a \in \mathcal{V}$, and $k_{c}, c \in T$ provide linear representations of the graph algebra and the extended Verlinde algebra respectively.)

We are thus in the position to use the results of sect. 5.1. The matrices $\left\{N_{a}, a \in T\right\}$ span a $\mathrm{C}$ - subalgebra $\mathcal{U}_{T}$ of $\mathcal{U}$, isomorphic to the extended Verlinde algebra. The latter is selfdual, hence the subalgebra $\mathcal{U}_{T}$ coincides with its dual algebra - a quotient of the dual algebra $\hat{\mathcal{U}}$. Indeed the subset $\hat{T}$ is identified with the set of weights in the class of identity $\{\rho\}$ and $\hat{\mathcal{U}}_{\hat{T}}$ is realised as a subalgebra of the $M$ matrices. The splitting of the set of exponents into the equivalence classes $\{\lambda\}$, each described by the decomposition of a representation of the extended algebra, agrees with the meaning of the equivalence relation in the sense of [25].

Let us rewrite the basic relation (3.13), using (5.8) and (5.13), as

$$
N_{\{\alpha\}\{\beta\}}^{\{\gamma\}} \sqrt{\frac{k_{c}}{k_{a} k_{b}}}=\sum_{\gamma \in\{\gamma\}} M_{\alpha \beta}^{\gamma} \sqrt{\frac{\hat{k}_{\gamma}}{\hat{k}_{\alpha} \hat{k}_{\beta}}}, \quad a=\{\alpha\}, b=\{\beta\}, c=\{\gamma\} \in T,
$$

identifying the equivalence classes $\hat{T}_{i}$ of $\hat{\mathcal{U}}$ with the representations $\{\lambda\}$ of the extended algebra. Then (5.16) can be seen as an expression for the structure constants $N_{\{\alpha\}\{\beta\}}^{\{\gamma\}}$ of the quotient algebra $\hat{\mathcal{U}} / \hat{\mathcal{U}}_{\hat{T}}$ in terms of the structure constants $M$ of $\hat{\mathcal{U}}$. The parameter $\hat{k}_{\{\gamma\}}=\sum_{\gamma \in\{\gamma\}} \hat{k}_{\gamma} / \sum_{\alpha \in\{\mathbf{1}\}} \hat{k}_{\alpha}=D_{\{\gamma\}}^{2}$ coincides with $k_{c}$ for $c \in T, c \equiv\{\gamma\}$. 


\section{Remarks}

1) Note that the relations of the type in (5.13), (5.14), follow from the general results of [25]; specific to the present application is the symmetry of the unitary matrix in the r.h.s. of (5.13), (5.14), and furthermore, the explicit realisation in (5.11).

2) Similarly there is a formula dual to (5.16), in which the $M$ - matrix elements are replaced by $N$ - matrix elements, the sum runs over $c \in T_{c}$, etc, and the l.h.s. is replaced by the structure constants of $\mathcal{U} / \mathcal{U}_{T}$, dual to the subalgebra $\hat{\mathcal{U}}_{\hat{T}}$, where $\hat{T}$ coincides with the identity class $\{\rho\}$. It leads to the dual counterparts of the relations (5.13), (5.14). However the analog of the extended $S$ matrix, which diagonalises the matrices representing the algebra $\mathcal{U} / \mathcal{U}_{T}$, (as well as the matrices $\left(M_{\alpha}\right)_{\beta}^{\gamma}, \alpha, \beta, \gamma \in$ $\{\mathbf{1}\})$ is not necessarily a symmetric matrix in general. Typically one has furthermore to enlarge the subset $\hat{T}$, i.e., to consider subalgebras of the $M$ - algebras, bigger than the identity subalgebra $\hat{\mathcal{U}}_{\{\rho\}}$, so that the automorphism (2.2) keeps $\hat{T}$ invariant. The structure constants of the factor algebra $\mathcal{U} / \mathcal{U}_{T}$, are not in general integer valued.

3) The relevance of the $C$-algebras for the study of the relation between graphs and nondiagonal modular invariants was first pointed out in [17]. The considerations above are to some extent inverse in spirit to what was done in [17] for the blockdiagonal cases of $s l(2)$ and $s l(3)$. Starting from the explicitly constructed graphs and $N$-algebras for these examples, it was there observed empirically that in all cases the extended fusion algebra is represented isomorphically by a subalgebra of the graph algebra $N$. This leads to the relation (5.14). It was furthermore argued, exploiting the approach of Bannai and Ito, that given a subalgebra of a type I graph algebra, described by a subset $T$ of the vertices, there is a natural dual partition of the set of exponents, which yields a type I modular invariant. In the present approach the knowledge of the modular invariant is assumed, from which it is derived that the graph algebra admits a subalgebra isomorphic to the extended Verlinde one. Furthermore the relation (3.13), which we traced to originate from the locality requirements in the field theory, provides an additional information and yields in particular the explicit general expression (5.11) for the subset $\left\{\psi_{a}^{(\lambda)}\right\}, a \in T$, in terms of the original $S_{\lambda \mu}$ and the extended $S_{\{\lambda\}\{\mu\}}$ modular matrices.

4) For completeness let us indicate what is the explicit set $T$ for each of the graphs in the figures of the present paper. For $s l(3)$, (see also [17]), the graphs of Fig 1 and 4 below have been drawn in such a way that the vertices of $T$ lie at the periphery of the graph: the respectively 4,6 and 3 external vertices form the set $T$. For the graph of Fig. 2, they are the vertices labelled 1 to 6 , and for that of Fig. 3, those labelled 1 to 10. 


\subsection{Other restrictions on the eigenvectors $\psi_{a}$}

According to property ix) in section 2 and to the discussion following eq. (2.20) the fundamental adjacency matrix $G_{1}$ always coincides with one of the $N$ matrices, $\left(G_{1}\right)_{b}^{c}=$ $N_{a_{f} b}^{c}$. This enlarges the set of $|T|$ explicit 1 - dim linear representations of the $M$ - algebra in (5.6), adding a new one. Indeed we have $\frac{\psi_{a_{f}}^{(\lambda)}}{\psi_{1}^{(\lambda)}}=\gamma_{1}^{(\lambda)}=\frac{S_{\hat{\Lambda}_{1}+\rho \lambda}}{S_{\rho \lambda}}$, cf. 2.16), and since the denominator $\psi_{1}^{(\lambda)}$ is known from (5.12), this determines $\psi_{a_{f}}^{(\lambda)}$ and gives for the corresponding eigenvalues of $M_{\lambda}$ the explicit expression

$$
\frac{\psi_{a_{f}}^{(\lambda)}}{\psi_{a_{f}}^{(\rho)}}=\frac{\gamma_{1}^{(\lambda)}}{\gamma_{1}^{(\rho)}} \sqrt{D_{\{\lambda\}} D_{\lambda}}=\frac{S_{\hat{\Lambda}_{1}+\rho \lambda}}{S_{\hat{\Lambda}_{1}+\rho \rho}} \sqrt{\frac{D_{\{\lambda\}}}{D_{\lambda}}}
$$

Now given (5.17) we add to the system (3.13) the equations

$$
\frac{\psi_{a_{f}}^{\left(\lambda_{1}\right)}}{\psi_{a_{f}}^{(\rho)}} \frac{\psi_{a_{f}}^{\left(\lambda_{2}\right)}}{\psi_{a_{f}}^{(\rho)}}=\sum_{\lambda_{3} \in \operatorname{Exp}} M_{\lambda_{1} \lambda_{2}}^{\lambda_{3}} \frac{\psi_{a_{f}}^{\left(\lambda_{3}\right)}}{\psi_{a_{f}}^{(\rho)}} .
$$

Similarly the complex conjugated eigenvector $\psi_{a_{f}}^{\left(\lambda_{1}\right) *}$ is expressed through the eigenvalues of the matrix $G_{n-1}$. As an illustration of the application of (5.17), (5.18), consider the example $\widehat{s l}(3)_{9} \subset \widehat{E}_{6_{1}}$ with modular invariant

$$
Z_{\widehat{s l}(3)_{9}}=\left|\chi_{1,1}+\chi_{1,10}+\chi_{10,1}+\chi_{5,5}+\chi_{2,5}+\chi_{5,2}\right|^{2}+2\left|\chi_{3,3}+\chi_{6,3}+\chi_{3,6}\right|^{2}
$$

Accounting for (4.2), 4.3), the system (3.13) reduces in this case to 3 independent equations for 6 unknown matrix elements $M_{(5,5)(5,5)}^{(5,5)}, M_{(5,5)(5,5)}^{(2,5)}, M_{(3,3)_{+}(3,3)_{+}}^{(3,3)_{-}}, M_{(3,3)_{+}(3,3)_{+}}^{(3,6)_{-}}$, $M_{(3,3)_{+}(3,3)_{-}}^{(5,5)}, M_{(3,3)_{+}(3,3)_{-}}^{(2,5)^{-}} \cdot\left(\right.$ Here $\left((3,6)_{ \pm}\right)^{*}=(6,3)_{\mp}$, etc.; \pm correspond to the representations $(2,1,1,1,1,1),(1,1,1,1,2,1)$ of $\widehat{E}_{6_{1}}$. Since the extended fusion algebra is of type $\mathbb{Z}_{3}$, i.e., any product contains only one representation, the summation in (5.18) runs effectively always over $\lambda_{3}$ within one class, $\left\{\lambda_{3}\right\}$. Then (5.18) adds 3 new equations for the 6 variables and thus allows to determine completely all $M$ matrices, and to reconstruct the graph denoted $\mathcal{E}_{1}^{(12)}$ in 17] (Fig. 4).

In exactly the same way one can solve also the case $\widehat{s l}(4)_{6} \subset \widehat{s l}(10)_{1}$ (see [26] for the expression of the modular invariant), thus exhausting, together with the example discussed in Appendix A, the cases $\widehat{s l}(4)_{k} \subset \hat{g}$, all described in (4.1).

More information on the eigenvectors can be obtained if we make the assumption that for any vertex $a$ belonging to the subset $T$, the graph of $G_{1}$ has only one edge starting 


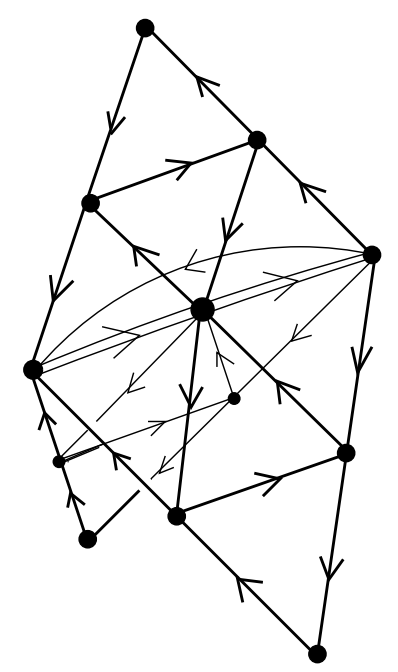

Fig. 4: The graph $\mathcal{E}_{1}^{(12)}$ corresponding to the conformal embedding $\widehat{s l}(3)_{9} \subset$ $\left(\hat{e}_{6}\right)_{1}$

from $a$ and one edge ending at $a$. This extra condition seems to be satisfied by graphs associated with conformal embeddings, although we do not know a proof of it. This implies that $G_{1} N_{a}=N_{b(a)}$ for any $a \in T$ and some vertex $b(a)$ and hence

$$
\frac{\psi_{b(a)}^{(\lambda)}}{\psi_{\mathbf{1}}^{(\lambda)}}=\frac{\psi_{a_{f}}^{(\lambda)}}{\psi_{\mathbf{1}}^{(\lambda)}} \frac{\psi_{a}^{(\lambda)}}{\psi_{\mathbf{1}}^{(\lambda)}}, \quad \forall a \in T .
$$

It is easy to see that all these vertices $b(a), a \in T$ form the class $T_{a_{f}}$. Using again (5.7), (5.8), we obtain new explicit eigenvalues of the $M$ matrices and hence new equations for their matrix elements.

The set of explicitly found $\psi_{b}^{(\lambda)}$ is furthermore enlarged by the complex conjugates of the above vectors, since they generate the class $T_{a_{f}^{\vee}} \cdot\left(T_{a_{f}}\right.$ and $T_{a_{f}^{\vee}}$ may coincide.)

Examining the graphs on Figs. 1,2,3 we see that (5.11), (5.17) and (5.20) (and their complex conjugates) together provide explicit analytic expressions for all eigenvectors $\phi_{a}$, $a \in \mathcal{V}$. These formulae are not sufficient for the graph on Fig.4 associated with the invariant (5.19) for which one recovers the eigenvectors corresponding to all but the three "central" vertices. These vertices have different values of the $\mathbb{Z}_{3}$ grading $\tau(a)$ introduced in section 2. This allows to express the moduli of the corresponding eigenvectors (and in particular the remaining unknown elements of the Perron-Frobenius eigenvector $\left.\psi^{(\rho)}\right)$ in terms of the known ones with the same grading, by exploiting the following relations:

$$
\begin{aligned}
& \sum_{b, \tau(b)=l}\left|\psi_{b}^{(\lambda)}\right|^{2}=\frac{1}{n}, \quad l=0,1, \ldots, n-1, \\
& \forall \lambda \in \operatorname{Exp}, \text { such that } \gamma_{p}^{(\lambda)} \neq 0, p=0,1, \ldots, n-1 .
\end{aligned}
$$


This follows from the basic property (2.13) of the matrices $G_{p}$ since

$$
\gamma_{p}^{(\lambda)} \sum_{b, \tau(b)=l} \psi_{b}^{*(\nu)} \psi_{b}^{(\lambda)}=\sum_{a, b ; \tau(b)=l} \psi_{b}^{*(\nu)}\left(G_{p}\right)_{b a} \psi_{a}^{(\lambda)}=\gamma_{p}^{(\nu)} \sum_{a, \tau(a)=l-p} \psi_{a}^{*(\nu)} \psi_{a}^{(\lambda)}
$$

for any $p=1,2, \ldots, n-1$. Choosing $\nu=\lambda$ and taking into account the first relation in (2.18a) we get in particular (5.21).

As the reader might have already noticed, the graphs drawn in Figs. 1-4 (all corresponding to conformal embeddings) possess some symmetry. Let us try to trace the origin of these symmetries.

Some of the symmetry comes from the $\mathbb{Z}_{2}$ group generated by the charge conjugation of the vertices, i.e., it reflects (2.14) and the first equality in (2.186).

Further restrictions on the set $\psi_{a}^{(\lambda)}$, and hence on the $N$ - matrices, result from the group $\Gamma_{\hat{g}}$ of automorphisms (to be denoted $\Sigma$ ) of the Dynkin diagram of $\hat{g}$, generated by automorphisms which do not fix the vertex corresponding to the affine root.

The induced action of $\Gamma_{\hat{g}}$ on the integrable weights of $\hat{g}$ can be lifted to the set of vertices $\mathcal{V}$. Indeed the subset $T$, being in one-to-one correspondence with the set of integrable weights of the extended algebra $\hat{g}$, remains invariant under the action of these automorphisms. Using (5.11) we have for $a \in T$

$$
\psi_{\Sigma(a)}^{(\lambda)}=e^{2 i \pi Q_{\Sigma}(\{\lambda\})} \psi_{a}^{(\lambda)}, \quad \lambda \in\{\lambda\}
$$

where the phase $Q_{\Sigma}(\{\lambda\})$ is specific to the extended algebra $\hat{g}$ and satisfies $Q_{\Sigma}(\{\rho\})=0$ : see e.g. [10] for details. Moreover (5.20) allows to extend this action to the elements of the class $T_{a_{f}}$, i.e., to define $\psi_{\Sigma(b(a))}^{(\lambda)}:=\gamma_{1}^{(\lambda)} \psi_{\Sigma(a)}^{(\lambda)}=\psi_{b(\Sigma(a))}^{(\lambda)}$, for any $a \in T$. In fact (5.23) extends to an arbitrary vertex $a$ as well, taking into account that $M_{\lambda \mu}^{\gamma}$ can be nonzero only if $Q_{\Sigma}(\{\gamma\})=Q_{\Sigma}(\{\lambda\})+Q_{\Sigma}(\{\mu\}) \bmod \mathbb{Z}$, since the same is true for the extended multiplicity $N_{\{\lambda\}\{\mu\}}^{\{\gamma\}}$. Furthermore it is not difficult to show that the invariance of $T$ under $\Sigma$ implies the invariance of any of the classes $T_{i}$, since due to the above definition we have $N_{a \Sigma(b)}^{\Sigma(c)}=N_{a b}^{c}$, and this is true in particular for $b \in T, a \sim c$. The equality (5.23) implies that for the fixed points of $\Sigma$ the elements $\psi_{a}^{(\lambda)}$ vanish identically whenever $Q_{\Sigma}(\{\lambda\})$ is nontrivial.

Combining (5.23) and (2.17) we have for any $a$ and $\lambda$ such that $\psi_{a}^{\lambda} \neq 0$,

$$
\frac{l}{n}(\tau(\Sigma(a))-\tau(a))=Q_{\Sigma}\left(\left\{\sigma^{l}(\lambda)\right\}\right)-Q_{\Sigma}(\{\lambda\}), \bmod \mathbb{Z} .
$$


The consistency of this relation restricts in general the automorphisms $\Sigma$ to some subgroup of $\Gamma_{\hat{g}}$.

\section{Examples:}

1. $k+3=12$ for $\operatorname{sl}(3)$. The extended algebra is $\hat{E}_{6}$ and the group $\Gamma_{\hat{g}}$ is isomorphic to $\mathbb{Z}_{3}$. The latter is realised by the fusion of the set of integrable representations of $\hat{E}_{6}$ for $k=1$. One has $Q_{\Sigma}(2,1,1,1,1,1)=-1 / 3=-Q_{\Sigma}(1,1,1,1,2,1)$ (see, e.g., 《10 ). The classes $T, T_{a_{f}}, T_{a_{f}^{\vee}}$, consist each of three elements, while the remaining three classes consist of one element, denoted, say, $a=4,8,12$, respectively - on Fig.4 these are the three interior vertices. This implies in particular that if $\lambda \in\left\{(3,3)_{ \pm}\right\}$, then $\psi_{a}^{\lambda}=0$ for $a=4,8,12$, since $\psi_{\Sigma^{l}(a)}^{(3,3)_{ \pm}}=e^{\frac{\nexists 2 \pi i l}{3}} \psi_{a}^{(3,3)_{ \pm}}=\psi_{a}^{(3,3)_{ \pm}}$.

2. The case $\widehat{s l}(5)_{3} \subset \widehat{s l}(10)_{1}$ : here (5.23) and (5.24) make sense for $\Sigma$ in the subgroup $\mathbb{Z}_{5} \subset \mathbb{Z}_{10}$, consisting of the even powers of the generating element $\Sigma^{1}$ defined as in (2.1) for $n=10 ; Q_{\Sigma^{2 s}}(\{\lambda\})=s t(\{\lambda\}) / 5$, where $t(\{\lambda\})$ is the standard $n$ - ality of the weight $\{\lambda\}$. In general the symmetry $(5.23)$ implies the invariance $\left(G_{p}\right)_{\Sigma(a) \Sigma(b)}=\left(G_{p}\right)_{a b}$. To check it in the case of Fig. 3 requires in particular the identification of vertices in $T$ with the integrable weights of $\widehat{s l}(10)_{1}$. The analysis of sect. 5.2 applied to this case reveals that the first six fundamental representations of $\operatorname{sl}(10)$ correspond to vertices labelled 1, 8, 5, 7, 4 and 6 in Fig 3; their conjugate are consistent with the involution $a \mapsto a^{\vee}$. It follows that $\Sigma^{2}$ maps cyclically vertices $1 \rightarrow 5 \rightarrow 4 \rightarrow 3 \rightarrow 2 \rightarrow 1$ and $6 \rightarrow 10 \rightarrow 9 \rightarrow 8 \rightarrow 7 \rightarrow 6$ as well as $11 \rightarrow 15 \rightarrow 14 \rightarrow 13 \rightarrow 12 \rightarrow 11$ and $16 \rightarrow 20 \rightarrow 19 \rightarrow 18 \rightarrow 17 \rightarrow 16$. Accordingly the graphs on Fig.3 are invariant under rotations by $2 \pi / 5$.

\subsection{The intertwiner}

We point out in this subsection a simple application of the general formula (5.11).

In [17] an intertwiner, which relates the adjacency matrices of the given graph and of the basic " $A$-" graph associated with $\operatorname{sl}(n)(n=2,3)$ with the same value of $h$, was constructed, namely

$$
\begin{aligned}
& N_{\hat{\Lambda}_{p}+\rho} V=V G_{p} \\
& V_{\gamma b}^{c}=\sum_{\omega \in \operatorname{Exp}} \frac{S_{\gamma \omega} \psi_{b}^{(\omega)} \psi_{c}^{(\omega) *}}{S_{\rho \omega}}, \quad b, c \in \mathcal{V}, \quad \gamma \in \mathcal{P}_{++}^{(h)} .
\end{aligned}
$$

It was furthermore observed that in all type I examples of $n=2,3$, the particular matrix elements $V_{\gamma 1}^{c}, c \in T$, encode the content of the blocks $\{\gamma\}$. This property of the 
intertwiner (5.25) may be now derived as a consequence of the explicit expression (5.11), namely 目

$$
V_{\gamma 1}^{c}=\sum_{\Omega} S_{c \Omega}^{*} \sum_{\omega \in \Omega} S_{\gamma \omega}=\operatorname{mult}_{\Gamma}(\gamma) \delta_{\Gamma c}, \quad c \in T
$$

i.e., $V_{\gamma 1}^{c}$ provides the multiplicity of $\gamma$ in $\Gamma \equiv c$. Here $S_{\gamma \omega}$ is the ordinary $S$ matrix whereas $S_{c \Omega} \equiv S_{\Gamma \Omega}$ refers to the extended one; we deviate from our usual notation, introducing $\Omega$ instead of $\{\omega\}$ for a representation of the extended algebra: this is to avoid confusion in cases where some $\omega$ belongs to several $\Omega$. The second equality in (5.26) is the standard consistency condition resulting from the modular properties of the character $\chi_{\Omega}=\sum_{\omega \in \Omega} \chi_{\omega}=\sum_{\omega \in \mathcal{P}_{++}^{(h)}} \operatorname{mult}_{\Omega}(\omega) \chi_{\omega}$.

More generally, if $b, c \in T$, we have

$$
V_{\gamma b}^{c}=\sum_{\Gamma \ni \gamma} \operatorname{mult}_{\Gamma}(\gamma) N_{\Gamma b}^{c}
$$

where $N_{\Gamma b}^{c}$ is the extended Verlinde multiplicity, and hence, at least for $b, c \in T, V_{\gamma b}^{c}$ are nonnegative integers (in particular zero for $\gamma \notin \operatorname{Exp}$ ). To get (5.27) insert for $\psi_{a}^{(\lambda)}$ the solution (5.11) and use again the consistency condition from the modular transformation of the characters in the form

$$
\sum_{\omega} \operatorname{mult}_{\Omega}(\omega) S_{\gamma \omega}=\sum_{\Gamma \ni \gamma} \operatorname{mult}_{\Gamma}(\gamma) S_{\Gamma \Omega}
$$

or, alternatively, insert (5.26) in $V_{\gamma b}^{c}=\sum_{a} V_{\gamma 1}^{a} N_{a b}^{c}$; for $b, c \in T$ this sum restricts to $T$. In the cases with no degeneracy of weights $\omega$, coming from different representations of the extended algebra, the sum in (5.27) reduces to one term and hence $V_{\gamma b}^{c}=\operatorname{mult}_{\{\gamma\}}(\gamma) N_{\{\gamma\} b}^{c}$.

\section{Conclusions.}

In this paper we have presented more evidence on the connection between conformal theories and graphs. This connection and a number of related facts that had so far remained empirical have received more support or have been proved to follow from natural assumptions.

Building upon our previous work on $s l(2)$ theories in which we had established some relations between data on the OPA and data relative to the graphs, we have extended

5 This has also been derived recently by Ocneanu 27] in a different context as reflecting the counting of "essential paths" on the graph. 
these relations to higher rank $\operatorname{sl}(n)$ theories. Our present work is restricted to conformal theories that we call of type I, for which the block diagonal form of the modular invariant signals the existence of an extended chiral algebra. Two plausible assumptions on the consistency of the operator algebra with this feature, namely eqs (3.9) (in the cases of conformal embeddings) and (3.10), have led us to the important relation (3.13) between fusion multiplicities, quantum dimensions and structure constants of the Pasquier algebra constructed out of the graphical data.

The validity of these assumptions and the practical importance of this relation (3.13) have been tested in a variety of cases of CFT associated with conformal embeddings. It has been shown that they allow in some cases to fully determine the graphs starting from the CFT. Thus new graphs have been obtained in a way that is much more systematical than the empirical procedures used so far. The relation (3.13) for the Pasquier algebra is presumably more general, as suggested by the example in App. B, and applies to all type I cases.

We have also reconsidered the use of the theory of $C$-algebras in connection with our problem. Merging this approach with the previous results provides a justification or a new perspective to results that had been obtained some time ago in [17]. Moreover an explicit general formula (5.11) has been derived for the components of the eigenvectors $\psi_{a}$, corresponding to the special set of vertices $T \ni a$, that are in one-to-one correspondence with the representations of the given extended theory.

What remains to do is to understand better the justification of the assumptions of sect. 3. Any progress in the solution of the general duality equations would eventually allow to extend the system of equations (3.13) and determine completely the Pasquier algebra, as was done in [3] in the $s l(2)$ case. Also cases with nontrivial multiplicities within a given representation of the extended algebra seem to present new situations that have just been tackled in App. A. Furthermore the extension of these results to theories of type II, i.e. that are not block diagonal, presents a challenge. In that respect the recent work of Ocneanu [27] seems to indicate that these theories may also be treated in a similar way and that there are interesting connections between the graphs pertaining to the pair of theories of type I and II obtained from one another by a twist.

Finally we notice that the new graphs found in this work yield new cases of infinite reflection groups, following the procedure of [28], and describe presumably patterns of solitons in $\mathcal{N}=2$ supersymmetric theories, as discussed by Cecotti and Vafa [29]. 


\section{Acknowledgements}

We would like to thank M. Bauer, A. Coste and P. Di Francesco for stimulating conversations. V.B.P. acknowledges the warm hospitality of the theory group at Saclay and the financial support of Commissariat à l'Energie Atomique, the hospitality of ASI, TU Clausthal, and a partial support of the Bulgarian Foundation for Fundamental Research under contract $F-404-94$.

\section{Appendix A. An example of nontrivial multiplicity}

In this Appendix we shall illustrate on the example $\widehat{s l}(4)_{4} \subset \widehat{s o}(15)_{1}$ the cases when an exponent $\lambda$ appears with a multiplicity higher than 1 in a given representation of the extended algebra.

The modular invariant associated with this embedding reads [30] :

$$
Z_{\widehat{s l}(4)_{4}}=\left|\chi_{111}+\chi_{151}+\chi_{123}+\chi_{321}\right|^{2}+\left|\chi_{115}+\chi_{511}+\chi_{212}+\chi_{232}\right|^{2}+\left|2 \chi_{222}\right|^{2} .
$$

The exponents in the three blocks correspond to the content in the decomposition of the three integrable representations $(1,1,1,1,1,1,1),(2,1,1,1,1,1,1)$, and $(1,1,1,1,1,1,2)$ of $\widehat{s o}(15)_{1}$ with quantum dimensions respectively 1,1 , and $\sqrt{2}$. They close on an Ising type fusion algebra. The representation $(2,2,2)$ of $\widehat{s l}(4)_{4}$ appears in $(1,1,1,1,1,1,2)$ with a multiplicity 2 . Thus unlike the previous examples the extended algebra does not distinguish the two fields to be associated with the exponent $(2,2,2)$. On the other hand if we identify these fields, i.e., look at them as two copies of one and the same field, the set of equations for the $M$ - matrices is checked to be inconsistent. To resolve the ambiguity of the representations $(2,2,2)$, which is a fixed point under the standard action of automorphism group $Z_{4}$, introduce two fields $((2,2,2) ; \pm)$ and define charge conjugation and the action of the $\sigma$ automorphism according to

$$
\sigma((2,2,2) ; \pm)=((2,2,2) ; \mp), \quad((2,2,2) ; \pm)^{*}=((2,2,2) ; \mp)
$$

i.e., each of the two fields is invariant under $\sigma^{2}$ only. For the other exponents keep the standard definitions of the charge conjugation and of $\sigma$. Then the last term in the modular invariant (A.1) can be interpreted as $\left|\chi_{(222) ;+}+\chi_{(222) ;-}\right|^{2}$. 
The definition of the charge conjugation implies that $M_{((2,2,2) ; \pm)((2,2,2) ; \mp)}^{(1,1,1)}=1$. With (A.2) the set of equations for the $M$ - matrices becomes consistent and we obtain using that $D_{(2,2,2)}=4(1+\sqrt{2}), \quad D_{(1,2,3)}=(1+\sqrt{2})^{2}$,

$$
\begin{gathered}
M_{(1,2,3)(3,2,1)}^{(1,2,3)}=\frac{1}{2}\left(\sqrt{D_{(1,2,3)}}-\frac{1}{\sqrt{D_{(1,2,3)}}}\right)=1, \\
M_{((2,2,2) ; \pm)((2,2,2) ; \pm)}^{(1,2,3)}=\frac{1}{2 D_{(1,1,1,1,1,1,2)}} \frac{D_{(2,2,2)}}{\sqrt{D_{(1,2,3)}}}=\sqrt{2}, \\
M_{((2,2,2) ; \pm)((2,2,2) ; \mp)}^{(1,2,3)}=\left(\frac{1}{2 D_{(1,1,1,1,1,1,2)}} \frac{D_{(2,2,2)}}{\sqrt{D_{(1,2,3)}}}-\frac{1}{\sqrt{D_{(1,2,3)}}}\right)=1 .
\end{gathered}
$$

The remaining matrix elements are either zero or determined from (4.2), (4.3), taking into account (A.2).

Diagonalising the $M$ matrices we obtain the fundamental matrices $G_{p}$ described by graphs depicted in Fig. 5
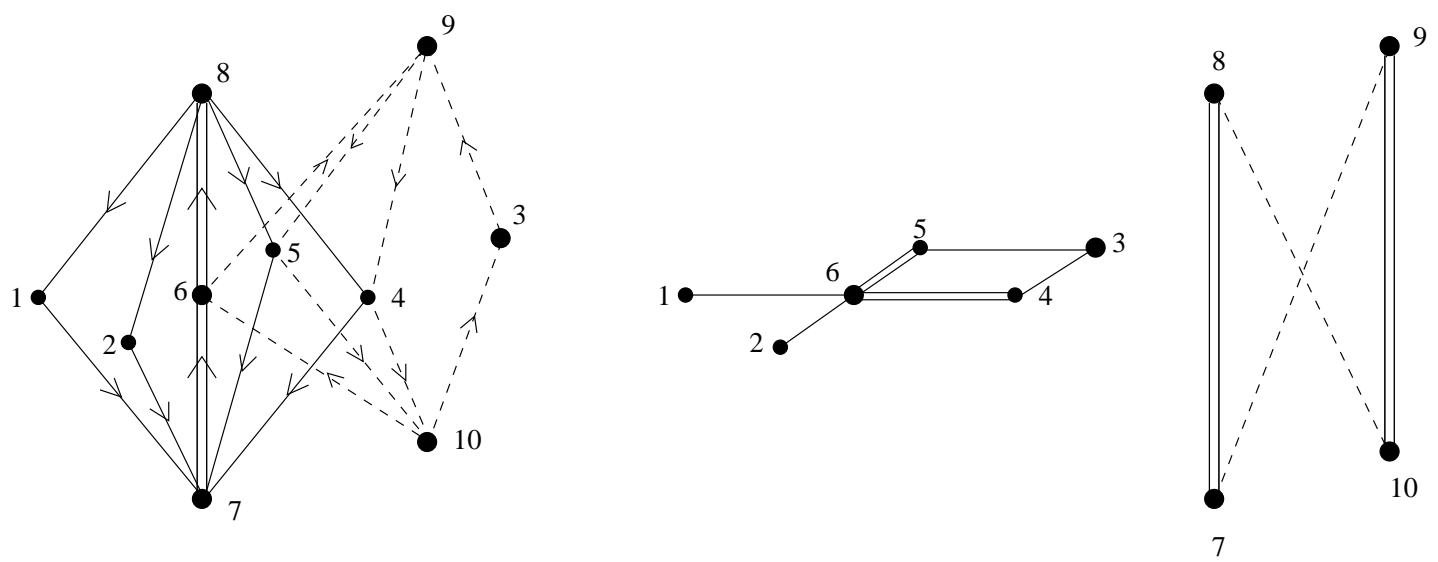

Fig. 5: The graphs of $G_{1}$ and $G_{2}$ corresponding to the conformal embedding $\widehat{s l}(4)_{4} \subset \widehat{s o}(15)_{1}$. The graphs should be viewed as having a $\mathbb{Z}_{2} \times \mathbb{Z}_{2}$ symmetry : $1 \leftrightarrow 2$ and $4 \leftrightarrow 5$. The broken lines denote edges carrying $\sqrt{2}$. The set $T$ is made of vertices 1,2 and 3 .

An unexpected feature is that these matrices have non integral entries !! This is reminiscent of what is obtained when one folds a $\mathbb{Z}_{2}$ symmetric graph: for example going from the $A_{2 n-1}$ Dynkin diagram to its $\mathbb{Z}_{2}$ quotient $B_{n}$, one finds that the (symmetrized) Cartan matrix of the latter contains some $\sqrt{2}$ entries. This suggests that the graphs above may be unfolded into graphs with $10+2=12$ vertices. 

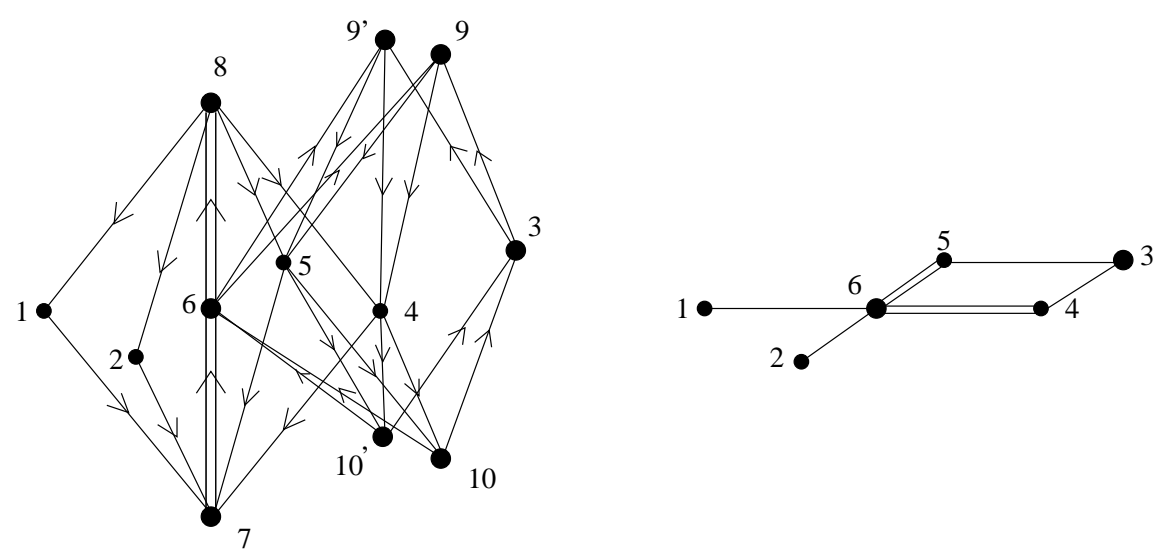

Fig. 6: The same graphs as in 5 after 'unfolding', i.e. duplication of vertices 9 and 10 into $9,9^{\prime}$, and $10,10^{\prime}$.

In fact if one returns to the modular invariant (A.1) and calls "exponents" the weights that label the diagonal terms of the sesquilinear form in the characters, one finds twelve such exponents. In that standpoint the exponent $(2,2,2)$ should come with a multiplicity 4 .

There is indeed a simple way of unfolding the graphs above. One just duplicates the vertices denoted 9 and 10 into $9,9^{\prime}$ and 10,10' and one replaces in $G_{1}$ and $G_{2}$ the entries $\sqrt{2}$ by $(1,1)$ or $\left(\begin{array}{l}1 \\ 1\end{array}\right)$ and the entry 2 by a $2 \times 2$ block $\left(\begin{array}{ll}1 & 1 \\ 1 & 1\end{array}\right)$, see the resulting graphs on Fig. 6. The exponent $(2,2,2)$ now appears 4 times. It seems, however, that it is impossible to make all the structure constants of the $M$ and $N$ algebras non negative.

It thus appears that in such a case with nontrivial multiplicities, there are two alternative attitudes:

- either one puts the emphasis on the extended algebra, i.e. the block structure of $Z$, with all the consequences that may be inferred on the $\psi$ 's, as discussed in the present paper, but at the expense of dealing with non integrally laced graphs, not covered by the considerations of sect. 2 ;

- or one follows the usual scheme of attaching an exponent to each diagonal term of $Z$, and constructs an integrally laced graph, but at the expense of a more complicated connection with the OPA.

\section{Appendix B. More on orbifolds of $s l(3)$}

This appendix is devoted to another series of CFT's to which our considerations apply: the so-called $\mathcal{D}$ series of orbifold $\widehat{s l}(3)$ theories for levels $k=0 \bmod 3$. In contrast with the 
case mentioned above in (2.21), they are endowed with a block modular invariant partition function 22

$$
Z=\frac{1}{3} \sum_{\substack{\lambda \in \mathcal{P}_{++}^{(k+3)} \cap Q \\ \text { ( }}}\left|\chi_{\lambda}+\chi_{\sigma(\lambda)}+\chi_{\sigma^{2}(\lambda)}\right|^{2}
$$

where we recall that $Q$ denotes the root lattice. The partition function (B.1) is as usually normalized in such a way that $\left|\chi_{\rho}\right|^{2}$ comes with a factor 1 . The term $\left|\chi_{\alpha_{0}}\right|^{2}$ associated with the fixed point $\alpha_{0}=\left(\frac{k+3}{3}\right) \rho$ of $\sigma(2.2)$ comes however with a multiplicity 3. Accordingly, we have to append an index $i=1,2,3$ when referring to the exponent $\alpha_{0}$.

For the $\widehat{s l}(3)$ orbifolds under consideration, the relevant graphs have been constructed long ago by Kostov [20]. They are obtained by an orbifolding procedure from the basic graphs of type $\mathcal{A}$ and the simplest example has been displayed in Fig. 1. They have a number of vertices equal to

$$
\frac{k}{2}\left(\frac{k}{3}+1\right)+3
$$

In the same way as in the case of the $D_{\text {even }}$ orbifolds of $\widehat{s l}(2)$ theories in which two subseries have to be distinguished, depending whether $\frac{k}{2}=0$ or $2 \bmod 4$, here we have to distinguish according to the value of $\frac{k}{3} \bmod 3$. This manifests itself in particular in the form of the extended $S$ matrix. That matrix satisfies (5.28), but this leaves some arbitrariness in the $3 \times 3$ block $S_{\left\{\left(\alpha_{0}, i\right)\right\}\left\{\left(\alpha_{0}, j\right)\right\}}$. The remaining elements are determined from (5.28), using that all exponents have $\tau=0$, and hence exploiting (2.17) gives $S_{\{\lambda\}\{\mu\}}=3 S_{\lambda \mu}$ for $\lambda, \mu \neq \alpha_{0}$, $S_{\{\lambda\}\left\{\left(\alpha_{0}, i\right)\right\}}=S_{\lambda \alpha_{0}}$ for $\lambda \neq \alpha_{0}$. From the Kac - Peterson formula for $S_{\mu \nu}$ it follows that $S_{\alpha_{0} \alpha_{0}}=-3 / h$ for $h=k+3=6 \bmod 9, S_{\alpha_{0} \alpha_{0}}=0$ for $h=0 \bmod 9, S_{\alpha_{0} \alpha_{0}}=3 / h$ for $h=3$ $\bmod 9$. Also note $S_{\alpha_{0} \rho}=3 / h$. The sum of the entries on any line or row in the $3 \times 3$ block in the extended matrix should be equal to $S_{\alpha_{0} \alpha_{0}}$ according to (5.28), i.e., accounting also for the symmetry we are left with 3 unknown parameters, to be determined e.g., by the unitarity condition, or the other conditions on the extended modular matrix. It appears that if we call $\kappa$ the integral part of $(k-3) / 9$, this $3 \times 3$ block has the following form

$$
\left(S_{\left\{\left(\alpha_{0}, i\right)\right\}\left\{\left(\alpha_{0}, j\right)\right\}}\right)=\frac{3}{h}\left(\begin{array}{ccc}
\frac{k}{3}-\kappa & -\kappa-1 & -\kappa-1 \\
-\kappa-1 & \frac{k}{3}-\kappa & -\kappa-1 \\
-\kappa-1 & -\kappa-1 & \frac{k}{3}-\kappa
\end{array}\right) .
$$

The charge conjugation of the classes $\left\{\left(\alpha_{0}, i\right)\right\}$ is assumed trivial, $\left\{\left(\alpha_{0}, i\right)\right\}^{*}=\left\{\left(\alpha_{0}, i\right)\right\}$, $i=1,2,3$ for all three subseries. 
From the relations between the $\mathrm{S}$ matrices of the original and of the extended theories, we deduce that $D_{\left\{\left(\alpha_{0}, i\right)\right\}}=\frac{D_{\alpha_{0}}}{3}$ and $D_{\{\lambda\}} \equiv D_{\lambda}$ otherwise. Then (3.13) reads:

$$
\sum_{l=0}^{2} M_{\lambda \mu}^{\sigma^{l}(\gamma)}=N_{\{\lambda\}\{\mu\}}^{\{\gamma\}}
$$

for $\lambda, \mu, \gamma$ all different from the fixed point $\alpha_{0}$;

$$
\sqrt{3} M_{\lambda \mu}^{\left(\alpha_{0}, i\right)}=N_{\{\lambda\}\{\mu\}}^{\left\{\alpha_{0}, i\right\}}
$$

for $\lambda, \mu$ different from the fixed point;

$$
\frac{1}{3} \sum_{l=0}^{2} M_{\left(\alpha_{0}, i\right)\left(\alpha_{0}, j\right)}^{\sigma^{l}(\gamma)}=M_{\left(\alpha_{0}, i\right)\left(\alpha_{0}, j\right)}^{\gamma}=N_{\left\{\left(\alpha_{0}, i\right)\right\}\left\{\left(\alpha_{0}, j\right)\right\}}^{\{\gamma\}}
$$

for $\gamma$ different from the fixed point (here we have used also (4.3) );

$$
M_{\left(\alpha_{0}, i\right)\left(\alpha_{0}, j\right)}^{\left(\alpha_{0}, l\right)}=\sqrt{3} N_{\left\{\left(\alpha_{0}, i\right)\right\}\left\{\left(\alpha_{0}, j\right)\right\}}^{\left\{\left(\alpha_{0}, l\right)\right\}} .
$$

Given the extended modular matrix one can compute by Verlinde formula the extended multiplicities and insert in these formulae.

All these relations as well as (5.11) have indeed been checked for the lowest representatives of the three subseries, $k=3,6,9$ and it is thus presumed that the equations (3.13) and (5.11) hold true in general for all these orbifold theories. Furthermore the equations (3.13) and (5.11) are expected to hold true for the general $\widehat{s l}(n)$ orbifolds of [20]. 


\section{References}

[1] V. Pasquier, Nucl. Phys. B285 [FS19] (1987) 162 ;

V. Pasquier, J. Phys. A20 (1987) 5707.

[2] P. Di Francesco and J.-B. Zuber, Nucl. Phys. B338 (1990) 602.

[3] V.B. Petkova and J.-B. Zuber, Nucl. Phys. B438 (1995) 347.

[4] A. Cappelli, C. Itzykson and J.-B. Zuber, Nucl. Phys. B280 [FS18] (1987) 445; Comm. Math. Phys. 113 (1987) 1 ;

A. Kato, Mod. Phys. Lett. A2 (1987) 585.

[5] T. Gannon, Comm. Math. Phys. 161 (1994) 233; The Classification of SU(3) Modular Invariants Revisited, hep-th 9404185.

[6] C. Itzykson, Nucl. Phys. (Proc. Suppl.) 5B (1988) 150 ;

P. Degiovanni, Comm. Math. Phys. 127 (1990) 71.

[7] T. Gannon and M.A. Walton, On the Classification of Diagonal Coset Modular Invariants, hep-th 9407055 .

[8] R. Dijkgraaf and E. Verlinde, Nucl. Phys. (Proc. Suppl.) 5B (1988) 87.

[9] G. Moore and N. Seiberg, Nucl. Phys. B313 (1989) 16; Comm. Math. Phys. 123 (1989) 177.

[10] T. Gannon, P. Ruelle and M.A. Walton, Automorphism Modular Invariants of Current Algebras, preprint LETH-PHY-3/95, US-FT/22-94, UCL-IPT-95/4, hep-th/9503141.

[11] E. Verlinde, Nucl. Phys. B300 [FS22] (1988) 360.

[12] F.M. Goodman, P. de la Harpe and V.F.R. Jones, Coxeter Graphs and Towers of Algebras, Springer-Verlag, Berlin (1989).

[13] N. Sochen, Nucl. Phys. B360 (1991) 613.

[14] D. Altschüler, J. Lacki and Ph. Zaugg, Phys. Lett. 205B (1988) 281.

[15] V.G. Kac and M.N. Sanielevici, Phys. Rev. D 37 (1988) 2231.

[16] V.G. Kac and M. Wakimoto, Adv. in Math. 70 (1988) 156.

[17] P. Di Francesco and J.-B. Zuber, in Recent Developments in Conformal Field Theories, Trieste Conference 1989, S. Randjbar-Daemi, E. Sezgin and J.-B. Zuber eds., World Scientific 1990 ;

P. Di Francesco, Int. J. Mod. Phys. A7 (1992) 407.

[18] V.A. Fateev and S.L. Lukyanov, Int. J. Mod. Phys. A3 (1988) 507.

[19] R. Brustein, S. Yankielowicz and J.-B. Zuber, Nucl. Phys. B313 (1989) 321.

[20] I.K. Kostov, Nucl. Phys. B 300 [FS22] (1988) 559.

[21] F.A. Bais and P.G. Bouwknegt, Nucl. Phys. B279 (1987) 561 ;

A.N. Schellekens and N.P. Warner, Phys. Rev. D 34 (1986) 3092.

[22] D. Bernard, Nucl. Phys. B288 (1987) 628.

[23] P. Christe and F. Ravanini, Int. J. Mod. Phys. A4 (1989) 897. 
[24] K.-H. Rehren, Ya.S. Stanev and I.T. Todorov, Characterizing invariants for local extensions of current algebras, preprint DESY 94-164 ESI 132, hep-th/ 9409509, Comm. Math. Phys. (1995) to appear.

[25] E. Bannai, T. Ito, Algebraic Combinatorics I: Association Schemes, Benjamin/Cummings (1984).

[26] A.N. Schellekens and S. Yankielowicz, Nucl. Phys. B327 (1989) 673.

[27] A. Ocneanu, communication at the Workshop Low Dimensional Topology, Statistical Mechanics and Quantum Field Theory, Fields Institute, Waterloo, Ontario, April 2630, 1995.

[28] J.-B. Zuber, Graphs and Reflection Groups, SPhT 95/089, hep-th 9507057, Comm. Math. Phys. to appear.

[29] S. Cecotti and C. Vafa, Comm.Math.Phys. 158 (1993) 569.

[30] A.N. Schellekens and S. Yankielowicz, Nucl. Phys. B334 (1990) 67. 